CERN-EP/2000-116

31 August, 2000

\title{
Performance of GEM detectors in high intensity particle beams
}

\author{
S. Bachmann, A. Bressan, B. Ketzer, M. Deutel \\ L. Ropelewski, F. Sauli \\ CERN, Geneva, Switzerland
}

A. Bondar, A. Buzulutskov, L. Shekhtman, A. Sokolov, A. Tatarinov, A. Vasil'ev

Budker institute of Nuclear Physics, Novosibirsk, Russia

\author{
S. Kappler \\ University of Karlsruhe, Germany \\ E. Schulte \\ University of Helsinki, Finland
}

\begin{abstract}
We describe extensive tests of Double GEM and Triple GEM detectors, including full size prototypes for the COMPASS experiment, exposed to high intensity muon, proton and pion beams at the Paul Scherrer Institute and at CERN. The measurements aim at detecting problems possible under these operation conditions, the main concern being the occurrence of discharges induced by beam particles. Results on the dependence of the probability for induced discharges on the experimental environment are presented and discussed. Implications for the application of GEM detectors in experiments at high luminosity colliders are illustrated.
\end{abstract}




\section{Introduction}

The gas electron multiplier (GEM) is a thin, metal clad polymer foil, perforated with a high density of holes [1]. Under application of a suitable potential difference between the two sides a high field is built up in the holes. A particle detector can be made inserting a GEM electrode between a drift cathode and a readout board with charge collection electrodes. A schematic view of such a detector is given in figure 1 . The structure is flushed by an appropriate counting gas. When an ionising particle traverses the detector it releases electron-ion pairs by ionisation. Electrons produced in the gap between the drift cathode and GEM drift into the holes where they are multiplied by the high electrical field, and transferred into the gap between GEM and the readout board. The collection of the electrons induces a detectable current on the pick-up electrodes.

The functional separation of the element for the gas amplification process from the pick-up electrodes allows full flexibility in the choice of the readout pattern. This permits a simple implementation of a two-dimensional readout of GEM detectors [2]. Two sets of parallel metal strips are engraved on each side of a thin polymer foil. The foil is glued to a thin support and then chemically removed in the spaces between the upper strips, exposing the bottom layer of strips; the electrons are collected by the strips in the two layers. To achieve an equal sharing of the charge, it is necessary to choose the width of the bottom strips larger than that of the upper strips. The geometry of different readout boards, their measured strip capacitance and the charge sharing between the two layers are given in table 1 . Figure 2 shows close-ups of a GEM foil and a two-dimensional readout board.

The charge extracted from a GEM can be collected directly, or transferred to another amplification device. A schematic view of multi-stage detectors, built with two and three cascaded GEM foils, is given in figure 3. It has been demonstrated [3], that with cascaded detectors a much higher gas gain is reached compared to single devices; moreover, for cascaded devices a given gain is achieved at much lower operating voltages, as the gas amplification process

\begin{tabular}{|c|c|c|c|c|c|}
\hline $\begin{array}{c}\text { pitch } \\
{[\mu \mathrm{m}]}\end{array}$ & $\begin{array}{c}\text { thickness } \\
{[\mu \mathrm{m}]}\end{array}$ & $\begin{array}{c}\text { width up } \\
{[\mu \mathrm{m}]}\end{array}$ & $\begin{array}{c}\text { width down } \\
{[\mu \mathrm{m}]}\end{array}$ & $\begin{array}{c}\text { capacitance } \\
{[\mathrm{pF} / \mathrm{cm}]}\end{array}$ & $\begin{array}{c}\text { charge sharing } \\
\text { up/down }\end{array}$ \\
\hline \hline 200 & 25 & 80 & 150 & 0.9 & 2.0 \\
\hline 400 & 50 & 150 & 350 & 1.2 & 1.5 \\
\hline 400 & 50 & 80 & 350 & 0.7 & 0.83 \\
\hline
\end{tabular}

Table 1

Properties of different geometries for the readout printed circuit board. They are made of Kapton with a thickness of $25 \mu \mathrm{m}$ 
is shared between several amplification stages. This results in a more reliable operation of the detector.

Use of multi-stage amplification devices was motivated by the observation that micro pattern gas detectors suffer from discharges triggered by heavily ionising particles crossing the detector [4]. Systematic laboratory measurements show that in the presence of heavily ionising particles all single stage micro pattern gas detectors can be operated only at a limited gain to avoid the occurrence of discharges [5]. This gain limit results in a marginal operation in modern high energy physics experiments. In this study we describe an investigation of these effects in realistic environments for high energy physics experiments.

\section{Detectors under investigation}

Results obtained with three detectors, two Double GEM and one Triple GEM, are described. All detectors have a two dimensional readout structure. The GEM foils and the 2D readout boards for all detectors were produced at the CERN workshops ${ }^{1}$. The geometry of the foils has been chosen after careful optimisation studies [6]: the Kapton thickness is $50 \mu \mathrm{m}$, the hole pitch $140 \mu \mathrm{m}$, and the hole diameter on the copper and Kapton centre are $70 \mu \mathrm{m}$ and $50 \mu \mathrm{m}$ correspondingly.

One of the two Double GEM detectors is made of small size $\left(10 \times 10 \mathrm{~cm}^{2}\right)$ GEM foils mounted in a general purpose assembly. The basic elements are two fibreglass frames with thin polymer windows, kept together by bolted screws. Gas tightness is guaranteed by the use of rubber joints. Signals from the pickup electrodes are carried by appropriately designed printed circuit boards, wire bonded to the readout electronics. To supply GEM with high voltage another coarse feed-through circuit is used. The GEM foils are stretched and glued to thin fibreglass frames. The detector consists of two cascaded GEM and a drift cathode; spacers define the gaps between these elements. The drift gap, $3 \mathrm{~mm}$ thick, defines the sensitive volume of the detector, the transfer and induction gap are $2 \mathrm{~mm}$ thick each. The readout has strips of $200 \mu \mathrm{m}$ pitch for both coordinates, the width of the upper and lower strips being $80 \mu \mathrm{m}$ and $150 \mu \mathrm{m}$, respectively. The separation of the two layers is $25 \mu \mathrm{m}$.

The active area of the Triple GEM detector is $10 \times 4 \mathrm{~cm}^{2}$. The 2D-readout PCB, $10 \mathrm{~cm}$ long, has the same geometry as the previous device. The thickness of the drift gap is $3 \mathrm{~mm}$, those of the transfer and induction gaps $1.6 \mathrm{~mm}$. The third detector tested is a Double GEM prototype for the COMPASS experiment, designed for use in a high energy experiment: large size and low mass [7]. Several prototypes have been built and tested in the laboratory. Their total size including all frames is $32 \times 32 \mathrm{~cm}^{2}$, the active area is $31 \times 31 \mathrm{~cm}^{2}$. Induction, transfer and drift gap have a thickness of 1,1 and $3 \mathrm{~mm}$, respec-

$\overline{1}$ Technology developed by A. Gandi and R. De Oliveira 


\begin{tabular}{|c|c|c|c|}
\hline & low fields & medium fields & high fields \\
\hline \hline $\begin{array}{c}\text { transfer fields } \\
{[\mathrm{kV} / \mathrm{cm}]}\end{array}$ & 2.0 & 4.0 & 4.0 \\
\hline $\begin{array}{c}\text { induction field } \\
{[\mathrm{kV} / \mathrm{cm}]}\end{array}$ & 4.0 & 4.0 & 7.0 \\
\hline
\end{tabular}

Table 2

Field configuration used during the test beam experiments. The exact values for the fields depend slightly on the applied GEM voltages.

tively. Mechanical rigidity and gas tightness is given by the use of two light honeycomb support plates, and $5 \mathrm{~mm}$ wide frames made of Stesalite, the latter defining the height of the gas gaps. This results in an average thickness for the complete detector module of less than $0.3 \%$ of a radiation length.

In order to maintain the distances between the GEM foils or the lower GEM foil and the readout board the use of spacers is almost mandatory. A $50 \mu \mathrm{m}$ thick, $1 \mathrm{~mm}$ wide Kapton strip, glued directly to the GEM foils, serves as spacer for the detector under test. In other prototype modules we use an epoxy-glass grid with a pitch of several $\mathrm{cm}$ and a wall width of $\sim 400 \mu \mathrm{m}$.

The GEM foils are segmented in 5 sectors to reduce the capacitance, and therefore the total energy in case of a discharge. All sectors are connected via resistors to a common power line except one sector in the central area; this has a diameter of $50 \mathrm{~mm}$, corresponding to the beam spot expected in the experiment. Independently powered, it allows to introduce a controlled dead area to avoid occupancy problems during high intensity runs. In the detector under test, one of the sectors was slightly weaker, probably caused by some epoxy glue accidently diffused to holes during construction, causing it to be more susceptible to discharges. The pitch of the pick-up electrodes is $400 \mu \mathrm{m}$, the upper strips have a width of $150 \mu \mathrm{m}$, the lower strips a width of $350 \mu \mathrm{m}$, separated by $50 \mu \mathrm{m}$ Kapton ridges. The electrodes of all detectors are connected to the high voltage supply by means of resistor networks as indicated in figure 3. The relative values of the resistors determine the fields for the transfer and induction gaps for given GEM voltages. Measurements have been performed with three different field configurations, subsequently labelled as low, medium and high fields. Table 2 shows the corresponding values for these configurations. For the drift field values between 2 and $4 \mathrm{kV} / \mathrm{cm}$ have been chosen. We measured the effective gain ${ }^{2}$ of the chamber by recording the signal current $I_{S}$ on the readout board and the counting rate $\mathrm{R}$ under irradiation with $9 \mathrm{keV}$ $\mathrm{X}$-rays. Given the number $n$ of electron-ion pairs per conversion $(\sim 320$ for

$\overline{2}$ Since the charge produced in the gas amplification process is collected by several electrodes (upper strips, lower strips and bottom side of the GEM foil) we define an effective gain per electrode (see for example [3]). 
$9 \mathrm{keV}$ photons), the gain can be calculated by the expression $M=I_{S} /(\mathrm{enR})$, with $e$ denoting the electron charge. The results for the COMPASS Double GEM prototype are plotted in figure 4. The counting gas is $\mathrm{Ar} / \mathrm{CO}_{2}$ in the proportions $70 / 30$. It is the same for all measurements presented. This prototype and first tests have been described in more detail elsewhere [8].

For individual strip readout the detector was connected to highly integrated amplifier cards based on the PreShape32 [9] and PreMux128 [10] chip to allow for an individual strip readout, having similar amplifier stages with a fast shaping time of about 50 ns. The PreShape32 allows to read out 32 adjacent strips and delivers parallel analogue output signals, while the PreMux128 chip multiplexes the signals from 128 strips to a serial output line. The data acquisition used to readout these chips was triggered by scintillators.

The noise of the readout electronics depends strongly on the capacitive load of the amplifier. The expected dependence is shown in figure 5 for the PreMux128 chip together with the values measured with the chip connected to the $10 \times 10 \mathrm{~cm}^{2}$ Double GEM and the $31 \times 31 \mathrm{~cm}^{2}$ COMPASS prototype. It shows the importance to minimise the capacitance on the readout structure. A recent redesign of the geometry lead to a reduction of the capacitance from the value of approximately $1.2 \mathrm{pF} / \mathrm{cm}$ for the readout board used for the present measurements to approximately $0.7 \mathrm{pF} / \mathrm{cm}$ (see table 1 ).

Figures 6 and 7 show the performance of the COMPASS prototype readout by the PreMux128 chip measured with fast electrons from a $\mathrm{Sr}^{90}$ source. The definition of the signal to noise ratio and efficiency, as well as the procedure for their estimation is given in section 3.2.

To detect discharges, two independent methods have been used. A simple method is to count overloads in the power supplies operated in the current limited mode, with a threshold set to $1 \mu \mathrm{A}$ above the nominal bias current through the resistor network. The other method uses current monitors ${ }^{3}$ to measure continuously the current drawn by the drift cathode; this current is sampled by means of a FlashADC ${ }^{4}$. Using offline-software the data are then analysed searching for spikes caused by discharges. The two methods gave essentially the same results.

\section{Experimental tests}

The COMPASS prototype has been tested for several weeks during August and September 1999 at CERN in the M2 beam line foreseen for the COMPASS experiment. In December 1999 the Double GEM, Triple GEM and the COMPASS prototype were tested in the $\pi \mathrm{M} 1$ beam at the Paul-Scherrer-Institut (PSI) in Villigen. This last exposure has been performed in the framework

3 Development of F. Beissel, RWTH Aachen, Germany

4 Development of F. Beissel, RWTH Aachen, Germany 
of systematic tests of micro pattern gas detectors in view of their use for the LHCb inner tracking system.

In this section results from those test experiments are presented.

\subsection{Measurements at the M2 beam at CERN}

The COMPASS prototype, equipped with the PreShape32 chip, has been exposed for several weeks to the muon and proton beam M2 at CERN. This beam has the SPS structure with a $2.45 \mathrm{~s}$ long spill every $14.4 \mathrm{~s}$. About $2 \times 10^{8}$ muons and respectively $2 \times 10^{6}$ protons per spill are delivered to the experimental area at maximum rate. With the COMPASS target installed, the environment corresponds to that expected as nominal running conditions for the final experiment. After an initial phase of measurements at moderate intensity to determine its working point, the detector was exposed to the full $\mu$ and proton beam intensity for several days. Figures $8 \mathrm{a}$ and $8 \mathrm{~b}$ show the pulse height spectra for both coordinates recorded applying equal voltages on the GEM foils. From the calibration of the gain, obtained by measurements of pulse height from an $\mathrm{Fe}^{55}$ source during the test beam period, a gain of about 6000 is deduced for these voltages. Despite the asymmetric charge sharing on the two coordinates, at that gain both spectra are well separated from the small noise peak visible on the left side of the histograms.

The principal aim of the experiment was to investigate whether the operation of Double GEM detectors under nominal beam conditions suffer from discharges, induced by the very high particle flux in the detector, or by secondary heavily ionising particles created in hadronic interactions upstream or in the detector itself. We monitored the occurrence of discharges as described in section 2. Figures $9 \mathrm{a}$ and $9 \mathrm{~b}$ show the time evolution of the intensity of the muon beam for about 15 hours. The same plot shows the occurrence of discharges. Figure 9a corresponds to data taken with only the central region switched on, and a potential difference of $440 \mathrm{~V}$ on each GEM, set well above the working point. The effective total gain at these voltage is $1.2 \times 10^{4}$, twice the gain expected for the operation of the detector in the experiment. Only three sparks have been recorded during the 15 hours of irradiation. Integrating the particle rate over the time a discharge probability of $3 \times 10^{-12}$ can be deduced for the central region at an effective total gas gain of $1.2 \times 10^{4}$. The discharge rate in the COMPASS prototype with only the outer sectors operational was higher (see figure $9 \mathrm{~b}$ ); during the same duration of irradiation we observed 13 discharges. This might be explained by the weakness of one sector due to the improperly handling during manufacturing as mentioned in section 2.

The discharge rate depends strongly on the GEM voltages and therefore on the gas gain. We measured the discharge rate as a function of the voltages applied to the GEM foils. The results are given in figure 10a for the $\mu$ beam 
and figure $10 \mathrm{~b}$ for the hadron beam. Despite the fact that the particle flux in the hadron beam is 100 times lower for muons, the absolute discharge rates per hour are comparable. It is also conspicuous that the discharge rates observed during the voltage scan is significantly higher than the discharge rate observed during the long term measurements described above. As the voltage scans were performed before the long term measurements, this effect might be explained due to some hardening of the detector during its exposure to the high intensity beam.

For the COMPASS experiment, even the highest measured discharge rate is still tolerable; nevertheless efforts have been undertaken to find conditions for spark free operation of the detectors in the course of the experiment [11].

\subsection{Measurements at the $\pi M 1$ beam at PSI}

\section{Operating conditions}

The $\pi \mathrm{M} 1$ beam at PSI provides either $215 \mathrm{MeV} / \mathrm{c} \pi^{-}$or $350 \mathrm{MeV} / \mathrm{c} \pi^{+}$, at rates up to $10^{7} \pi^{-} / \mathrm{s}$ and up to $5 \cdot 10^{7} \pi^{+} / \mathrm{s}$. This has been verified measuring the coincidence rate of two $5 \mathrm{~mm} \times 5 \mathrm{~mm}$ scintillators in the beam. The beam spot has a size of about $8 \times 10 \mathrm{~cm}^{2}$; at highest beam intensity, the flux in the centre is $8 \mathrm{kHz} / \mathrm{mm}^{2}$. While pions of both polarities at an energy of $300 \mathrm{MeV}$ are minimum ionising, the $\pi^{+}$beam is contaminated by protons of $63 \mathrm{MeV}$ with an energy loss of about $7.5 \mathrm{MeV} \mathrm{g}^{-1} \mathrm{~cm}^{2}[12]$. At maximum $\pi^{+}$intensity, the contamination is about $2 \%$. Due to the small range in matter mainly the first detector in the beam line is affected by these protons, for our set-up the COMPASS prototype.

The detectors have been operated for about 100 hours, in about equal proportion at low and high intensity. To estimate the working point of the Double GEM and Triple GEM detectors for the detection of minimum ionising particles their pulse height distribution has been measured. Due to diffusion, the charge produced in the amplification process in the GEM-foils is spread over several strips. Examples of spectra for the largest signal amplitude in a cluster are shown in figure 11a for the Double GEM and in figure 12a for the Triple GEM. To reconstruct the whole charge produced in the avalanche, the signal of all adjacent strips exceeding a threshold of two times their noise level are added. The resulting cluster charge distributions are shown in figure 11b and $12 \mathrm{~b}$. In the spectra, a small peak on the left side is visible, caused by events with no reconstructed cluster and corresponding to the noise. As an acceptance criterion, we have used the folowing condition:

$$
Q_{\text {cluster }}>5 \times \sigma_{\text {cluster }},
$$

where $\sigma_{\text {cluster }}$ is the noise defined as the quadratic sum of the noise of all strips assigned to the cluster. The ratio of the number of clusters fulfilling the 
acceptance criterium to the number of events in the measured distribution gives an estimate of the detection efficiency ${ }^{5}$. The dependence of the efficiency on the applied voltage is shown in figures 13a and 13b. The figures also provide the signal to noise ratio, defined as the most probable value of the distribution

$$
S / N=\frac{Q_{\text {cluster }}}{\sigma_{\text {cluster }}} .
$$

In the described configurations the efficiency reaches a constant value when the $S / N$ exceeds a value of about 20 .

Another important property to characterise a micro-pattern detector is the cluster width, determined by the charge cloud creating the signal on the strips of the detector. It affects the occupancy and the double track resolution. In GEM detectors, this width is dominated by the diffusion of the electrons through the drift, transfer and induction gaps [13]. Usually the cluster width is defined as the number of strips over threshold. An example of this distribution is given in figure 14 for the Double GEM. The drawback of this definition is its dependence on the noise performance of the readout electronics and the algorithm used to reconstruct the cluster. A better definition of the cluster width is the FWHM of a Gaussian fit to the measured charge profile. An example is also given in figure 14 and shows only a weak dependence on the operating parameters (see figure 15), while for the former definition the cluster width depends on the operating voltages.

The experimental determination of the double track resolution of micro-strip detectors is difficult as it would require a precise external tracking system. Alternatively, the following software procedure has been followed to get an estimate of the double track resolution that can be achieved: two independent events, containing a cluster fulfilling the above mentioned definition, are selected in the offline analysis. The entries for the strips assigned to the cluster of one event are superimposed to the second event, with a distance adjusted as multiple of the detector pitch. By this method, artificial events containing double tracks are generated. The events are then analysed; if a cluster is found, it is determined whether it contains multiple peaks as explained in figure 16. If multiple peaks are found, the cluster is broken into two clusters, and it is checked if both fulfill the acceptance criteria. This method creates fake hits only in a very small fraction $(1 \%-2 \%)$ of events. Using the event sample containing double tracks, the probability that tracks are resolved is determined; the result is shown in figure 17, as a function of the distance between the clusters. As it can be seen, two tracks separated by about $500 \mu \mathrm{m}$, corresponding approximately to the FWHM of the cluster width, are resolved with a probability of about $50 \%$. This result confirms that the $400 \mu$ m readout pitch chosen for the detectors in the COMPASS experiment corresponds to

\footnotetext{
5 A better estimate of the efficiency requires a track definition by an external system. The value of the efficiency defined by the described method underestimates the real efficiency as some fake triggers from the scintillators cannot be suppressed.
} 
an optimum value, as it minimises the number of electronic readout channels while maintaining the intrinsic double track resolution of the detector. In addition, the lower strip count of the clusters results in a better $S / N$, allowing the detector to operate at lower gain.

The discharge probability has also been measured in this environment as a function of the GEM voltages for the medium and low field configuration. The results are shown in figures $18 \mathrm{a}$ and $18 \mathrm{~b}$. At the working point of the detector they indicate a probability of one discharge per $\sim 10^{9}$ particles passing through the detector. No dependency of the discharge probability on the particle flux has been observed.

The measured discharge probabilities of the COMPASS prototype are given in figure 19. While the discharge probabilities measured at particle rates between $10^{6}-10^{7} \pi^{-} / s$ are compatible to the results from the smaller size Double GEM, those obtained at the maximum intensity of $5 \times 10^{7} \pi^{+} / \mathrm{s}$ show significantly higher values. We explain that effect by the presence of a large amount of protons in the $\pi^{+}$beam (see section 4 ).

The results obtained with the Triple GEM detector are summarised in figures 20 to 22 . The dependence of gain on the voltage shown in figure 20a has been measured using the internal calibration input of the PreMux128 chip. The cluster finding algorithm is the same as used for the Double GEM. To calculate efficiency, a cut at 10 ADC bins on the cluster charge distribution shown in figure $12 \mathrm{~b}$ is applied. The measured efficiency and signal to noise ratio as a function of gain for both coordinates are given in figure 20b. Full efficiency for both coordinates is reached at unusually high gas gain of about $1.5 \times 10^{4}$; this is probably caused by a large external noise of unknown origin observed during the experiment. The cluster width of the Triple GEM is comparable to that measured for the Double GEM (see figure 21), the introduction of a third GEM foil being compensated by the reduced transfer and induction gaps.

At the operating voltage of $380 \mathrm{~V}$ on each GEM foil, we measured a discharge probability of $10^{-11}$, two order of magnitudes lower as compared to the Double GEM. This is due to the lower operating voltage, and therefore lower gain for a single GEM foil, making it less susceptible to discharges [11]. The dependence of the discharge probability on the GEM voltage can be seen from figure 22. Using an optimised readout board with respect to equal charge sharing and a lower strip capacitance (see section 2), full efficiency can be probably reached at an operating voltage of $370 \mathrm{~V}$ on each GEM. At that voltage we did not observe any discharge during an exposure time of more than 12 hours at maximum beam intensity. It has been observed that the occurrence of discharges in micro pattern gas detectors may damage the electrode structure, leading in the worst case to a short circuit between anode and cathode [4]. In addition the energy released in a discharge might flow to ground through the amplifier of the readout electronics. Unless the amplifier is protected, it can be destroyed. From our present experience GEM foils appear very robust against damages caused by discharges; during the test beam exposures, the detector could withstand without damages thousands of discharges. In former 
laboratory measurements, however, we observed damages of the PreShape32 readout chip connected to Double GEM detectors, caused by discharges in the detector. For that reason a careful analysis of possible damages of the readout electronics has been performed during the test beam experiment. It was made using data from the Double GEM and Triple GEM equipped with the PreMux128 chip, having a better input protection compared to the PreShape32. In figure 23a the noise measured at the beginning of the run is shown; dead electronics channels are clearly visible due to their high noise. It has been also verified that dead electronic channels identified that way correspond to holes in the beam profile. Damages of electronic channels occurred in the course of the test beam experiment can be identified by a comparison of the electronic noise before and after the runtime. Figure 23b shows the noise measured at the end of the run time, and figure $23 \mathrm{c}$ the difference in noise at the beginning and the end of the run time, excluding the initial dead channels. As no channel exhibits a significant increase in noise, it can be concluded that no damage to the readout electronics has been observed during the test beam experiment. For the Triple GEM detector also no sign of damages has been observed.

\section{Discussion}

The discharge probability of Multi GEM detectors shows a strong dependence on the radiation environment, e.g. the particle type, flux and energy. The results seem consistent with the idea that discharges are triggered by heavily ionising tracks

releasing an exceptionally high amount of electron-ion pairs in the active gas volume. This can be clearly seen in the results obtained with the COMPASS prototype. For this detector, the lowest discharge rate has been measured in the muon beam at M2, consisting essentially only of minimum ionising particles. For the proton beam in the same experimental area and the $\pi^{-}$beam at PSI comparable discharge rates have been determined, despite the much lower beam intensities. These beams also consist of minimum ionising tracks, but occasional heavily ionising particles might be produced by nuclear interactions as secondary particles. The highest discharge rate has been measured in the $\pi^{+}$beam at PSI. This beam has a fraction of $2 \%$ of protons at a kinetic energy of $63 \mathrm{MeV}$ supplementary to the beam intensity of $5 \times 10^{7} \pi^{+} / \mathrm{s}$, with a most probable energy loss of about $15 \mathrm{keV} / \mathrm{cm}$ and a long tail to higher energy losses. This value exceeds that of minimum ionising particles by a factor of 6 (see for example [12]). The mechanism which is most probably responsible for the breakdown is the transition from avalanche to streamer, followed by a discharge between anode and cathode, as has been extensively studied in gas detectors working in parallel plate mode ([14] - [16]): when a traversing particle releases a sufficiently high number of electron-ion pairs in the active gas 
volume of the detector the total charge created in the subsequent charge amplification process might exceed a certain value, corresponding to the Raether limit for the formation of a streamer in the avalanche. The streamer produces an ionised and therefore conductive channel between the anode and cathode. Along that channel the discharge takes place. The discharge probability observed in the Triple GEM is two orders of magnitude lower compared to the Double GEM; this suggests that the absolute value of charge corresponding to the Raether limit is not a fixed value, but depends on the specific operating conditions like the voltage applied to the GEM, an hypothesis already discussed in [5]. This result encourages a further optimisation of Multi GEM detectors in order to minimise their discharge probability for the operation in harsh environments. Several approaches seem promising. For the test beam measurements, the operating voltages where chosen to distribute the gain equally to all GEM foils; however, former results indicate that an asymmetric operation of the detector can improve its performance [5]. Using a different gas mixture might be another way to quench discharges, as the used gas mixture of Argon $/ \mathrm{CO}_{2}(70 / 30)$, though very convenient being not flammable, is known to be unstable. It should be also mentioned that the optimisation of the parameters of the GEM foils itself has been done to reach a maximum gas gain under irradiation of $\mathrm{X}$-rays [6]. It is not excluded that for the operation in presence of heavily ionising particles another choice of GEM parameters like the hole diameter yields better results.

The discharge probability shows a strong dependence on the gas gain of the detectors; it also seems to follow a statistical process, instead of being a threshold effect. The sensitivity of the discharge probability on environmental factors like the particle flux, the particle type and energy as well as the statistical nature underlying the discharge mechanism suggests that in the course of an experiment the occurrence of discharges cannot be completely excluded. The use of micro pattern gas detectors demands therefore a robust technology, not suffering from the development of discharges. Multi-GEM detectors seem to fulfil that demand: even though the detectors experienced several thousands

of discharges in the course of the discussed test beam experiments, damages were not observed neither to the GEM foils nor to the readout electronics.

\section{Summary and conclusion}

We performed extensive tests of several Multi GEM detectors in different test beam-lines at the Paul Scherrer Institute and at CERN, including high intensity muon, proton and pion beams. Two Double GEM detectors, one being a prototype for the COMPASS experiment, and a Triple GEM detector were tested. All detectors have a two-dimensional readout structure. The aim of the tests was to find out whether the detectors suffer from the operation 
in these environments, the main concern being the occurrence of discharges induced by beam particles.

The measurements show that the probability of a discharge depends strongly on the environmental factors like the particle flux, particle type or energy. Nevertheless the results are consistent with the idea that the dominant factor responsible for discharges is exceeding the so-called Raether limit: this can be reached when a particle traversing the detector releases an exceptionally high number of electron-ion pairs. During the experiments, the detectors proved to be robust enough to withstand several thousand discharges without damages; also, the PreMux128 chip connected to the detectors did not suffer from discharges. The discharge rates measured indicate that Double GEM detectors, even if used with two-dimensional readout demanding higher gain, can be used for most applications in high energy physics. In case of more stringent demands a further optimisation to increase the limits of these devices caused by discharges seems to be possible.

To increase even further the reliability of GEM detectors in high energy physics experiments, the application of Triple GEM detectors might be envisaged; in this case an operation at a gain of about $6 \times 10^{3}$, sufficient for the fully efficient detection of minimum ionising particles with a two-dimensional large area detector, was possible without any discharge. From this result we deduce an upper limit of the discharge probability of $2 \times 10^{-12}$ for $350 \mathrm{MeV} \pi^{+}$.

\section{References}

[1] F. Sauli, Nucl. Instrum. Methods A386 (1997) 531.

[2] A. Bressan et al., Nucl. Instrum. Methods A425 (1999) 254.

[3] S. Bachmann et al., Nucl. Instrum. and Methods A438 (1999) 376.

[4] B. Schmidt, Nucl. Instrum. and Methods A419 (1998) 230.

[5] A. Bressan et al., Nucl. Instrum. Methods A425 (1999) 262.

[6] J. Benlloch et al., Nucl. Instrum. Methods A419 (1998) 410.

[7] G. Baum et al, Proposal for a Common Muon and Proton Apparatus for Structure and Spectroscopy, CERN/SPLSC 96-14 (1996).

[8] S. Bachmann et al., Proc. 5th International Conference on Position-Sensitive Detectors, London, 13-17 September 1999. Submitted to Nucl. Instr. and Methods.

[9] L.L. Jones, PreShape32 specification, version 1.0, RD20 internal document, 1993.

[10] L.L. Jones, PreMux128 specification, version 2.3, RD20 internal document, 1995. 
[11] S. Bachmann et al. Discharge mechanisms and prevention in the Gas Electron Multiplier, in preparation.

[12] W.R. Leo, Techniques for Nuclear and Particle Physics Experiments, Springer Verlag (1992).

[13] A. Bressan et al., Nucl. Instrum. and Methods A425 (1999) 262.

[14] J.M. Meek, Physical Review 57 (1940) 722.

[15] P. Fonte et al., Nucl. Instrum. Methods A305 (1991) 91.

[16] P. Fonte, IEEE Trans. Nucl. Sci. 43 (1996) 2135. 


\section{List of Figures}

1 Schematic view of a Single GEM detector with two-dimensional projective readout board.

2 Close-up of a) a GEM foil, b) a two-dimensional readout board.

3 Schematics of a Double GEM and a Triple GEM.

4 Effective gain measured on the upper coordinate for the COMPASS prototype determined with X-rays. The field configurations are given in table 2 .

$5 \quad$ Noise performance of the PreMux128 chip. The triangles correspond to measurements, the solid line correspond to the specification given for that chip.

6 Signal to noise ratio measured for the upper coordinate of the COMPASS prototype with fast electrons from a $\mathrm{Sr}^{90}$ source.

7 Efficiency measured for the upper coordinate of the COMPASS prototype with fast electrons from a $\mathrm{Sr}^{90}$ source. The efficiency is measured with respect to a trigger signal from scintillators.

8 Pulse height spectrum and noise from the Compass prototype for the a) upper b) lower coordinate, measured in the M2 $\mu$-beam.

9 Beam intensity monitored at the $\mu$ beam at M2.

a) Only the central region of the detector was switched on.

b) The central region of the detector was switched off.

The occurrence of discharges is indicated.

10 Discharge rate measured with the COMPASS prototype in the M2 a) muon beam; b) proton beam.

11 Double GEM results from tests at PSI: a) Charge collected by the strip with the largest signal. b) Total charge in a cluster.

12 Triple GEM results from tests at PSI: a) Charge collected by the strip in the cluster with the largest signal. b) Total charge in a cluster. 
13 Signal to noise and efficiency for the upper coordinate of the $10 \mathrm{~cm} \times 10 \mathrm{~cm}$ Double GEM measured at PSI: a) medium field configuration; b) low field configuration.

14 Cluster width distribution measured at PSI with the $10 \mathrm{~cm} \times$ $10 \mathrm{~cm}$ Double GEM.

15 Cluster width as a function of the GEM voltage.

16 Cluster definition used for the analysis of the double track resolution: Case $\mathrm{A}$ and $\mathrm{B}$ will be resolved by the cluster algorithm while for case $\mathrm{C}$ a single cluster will be reconstructed.

17 Computed double track resolution.

18 Discharge probability and efficiency for the Double GEM at a) medium and b) low fields fields. The measurements were performed at the PSI $\pi \mathrm{M} 1$ beam.

19 Discharge probability for the COMPASS prototype measured at PSI.

20 Performance of the Triple GEM: a) gain, $\Delta \mathrm{V}_{G E M}$ is the voltage applied to each GEM foil; b) signal to noise and efficiency as a function of gas gain in the Triple GEM measured in the $\pi \mathrm{M} 1$ beam at PSI.

21 Cluster width in the Triple GEM measured at the PSI $\pi \mathrm{M} 1$ beam.

22 Discharge probability for the Triple GEM measured at the PSI $\pi \mathrm{M} 1$ beam.

23 Channel noise before (a) and after (b) the PSI runs; c) is the difference between a) and b), with the initial dead channels excluded. 


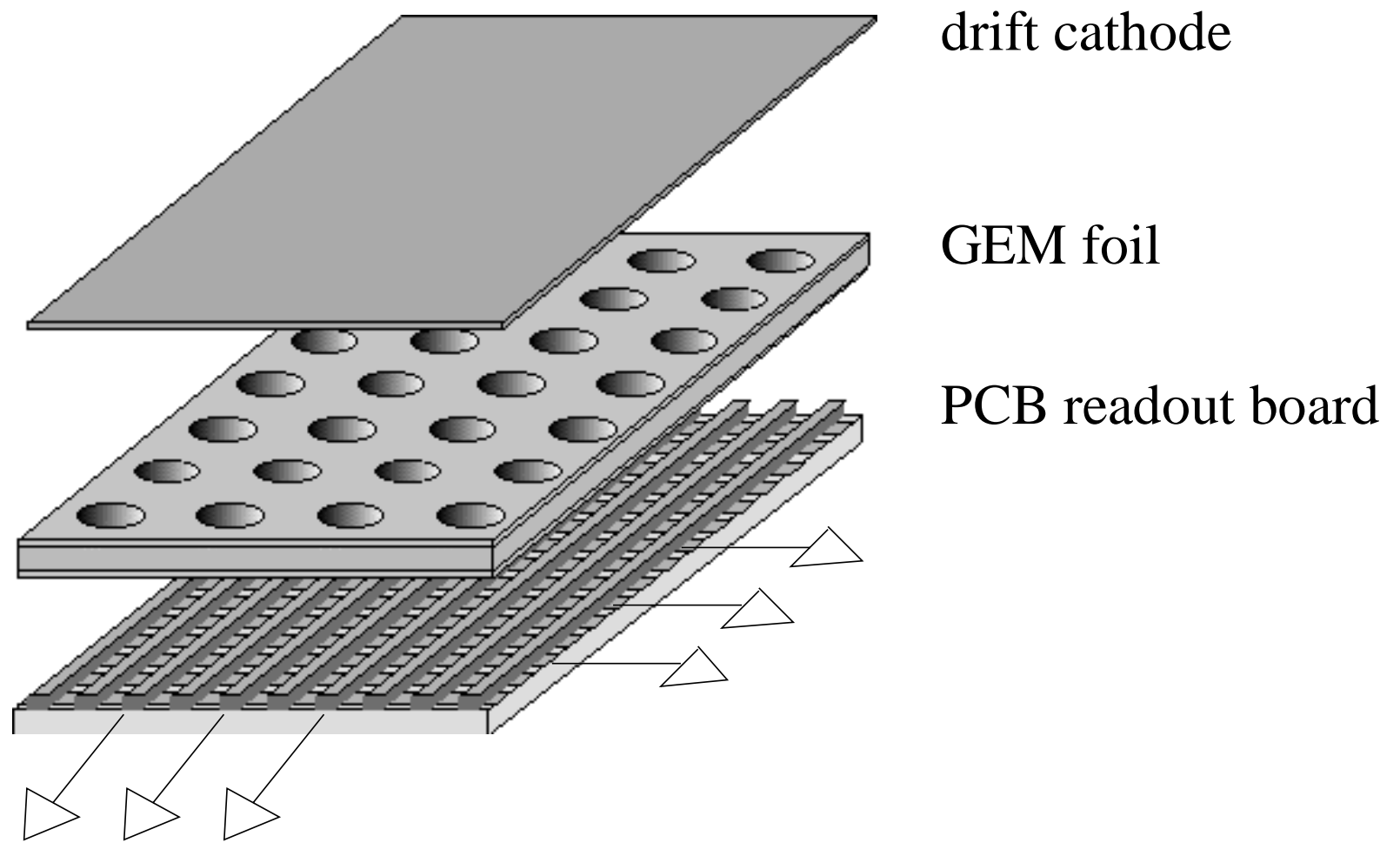

Figure 1. 
a)

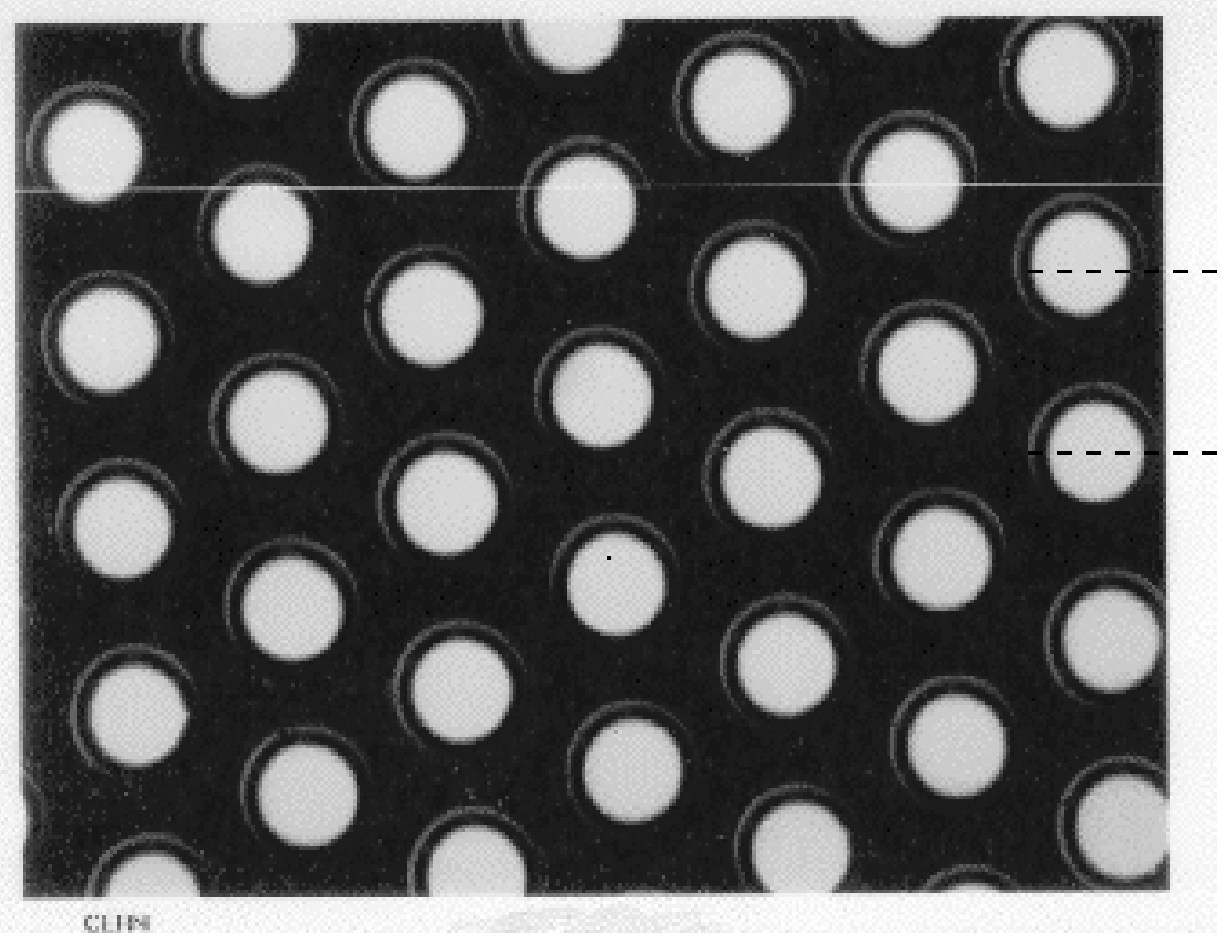

b)

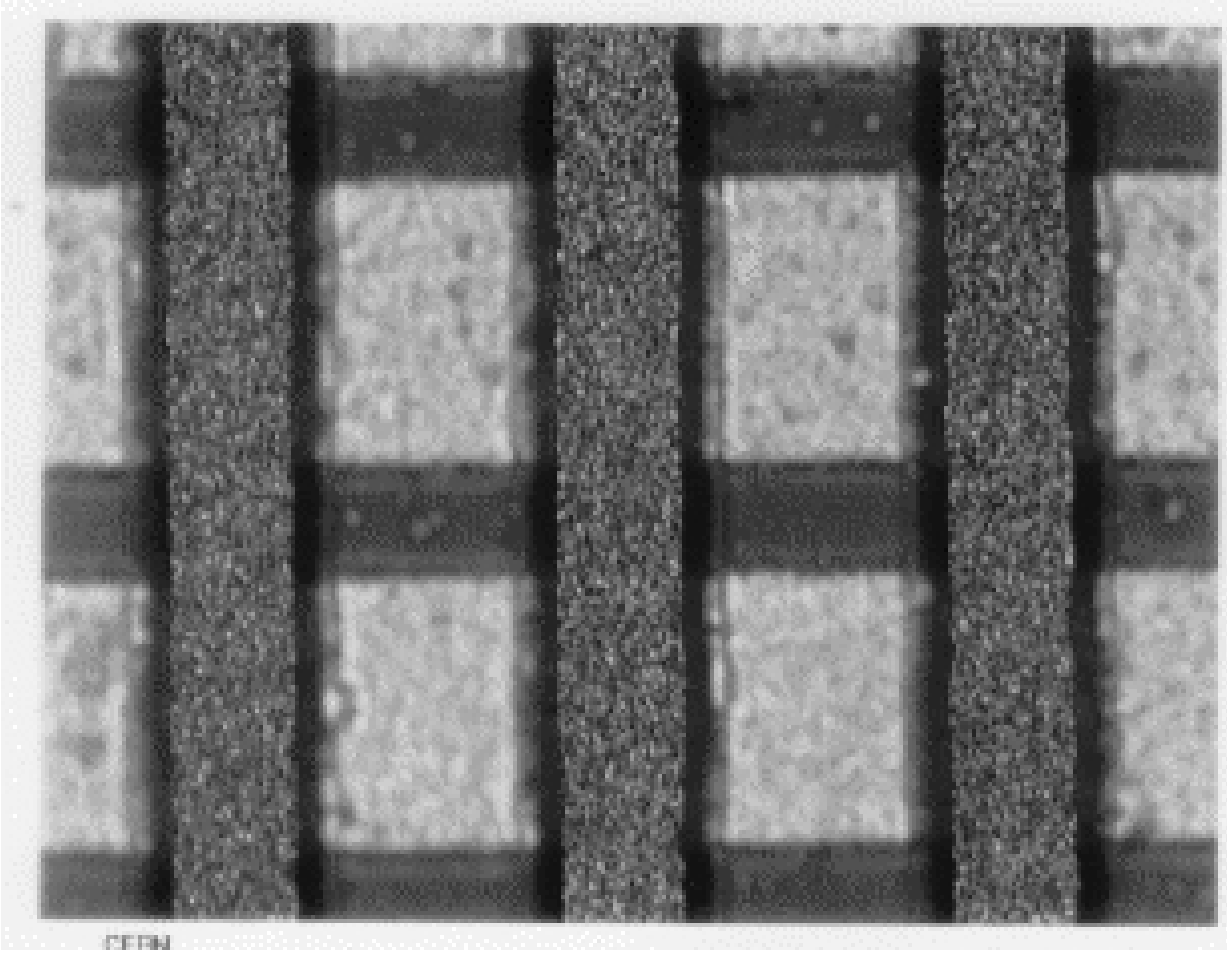

Figure 2. 


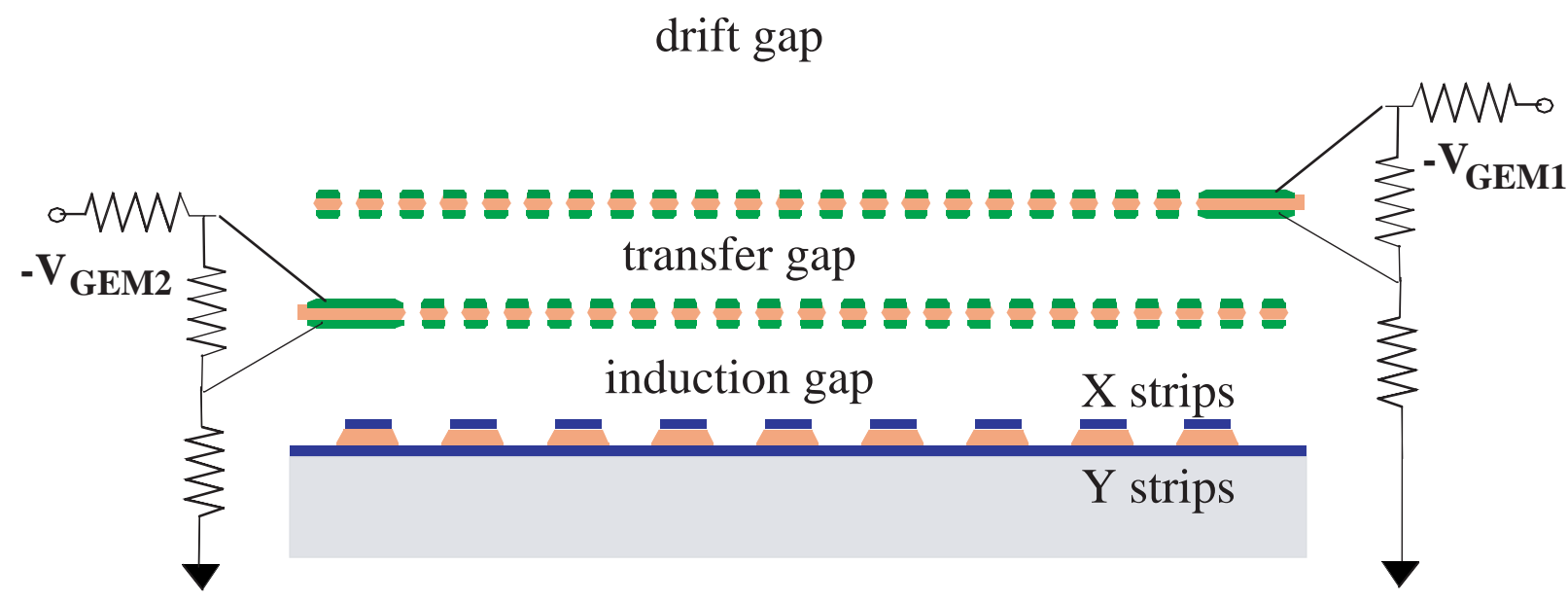

$-V_{D}$ o- WW

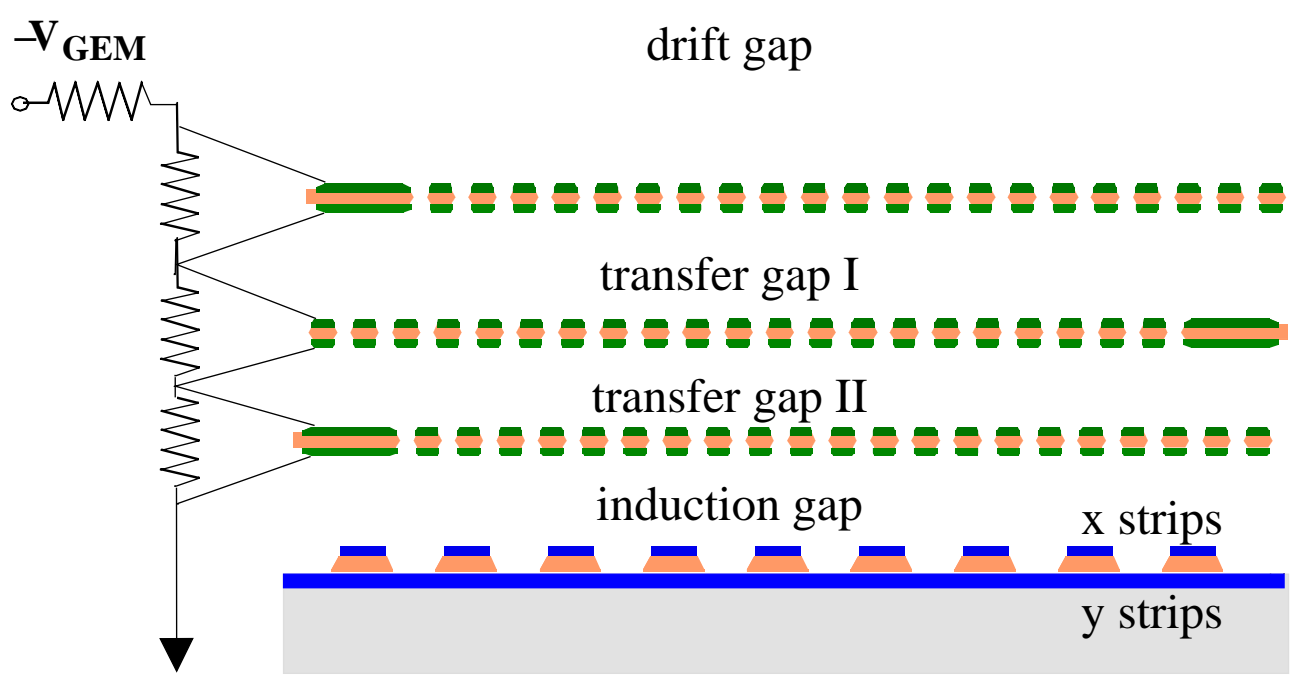

Figure 3. 
COMPASS prototype

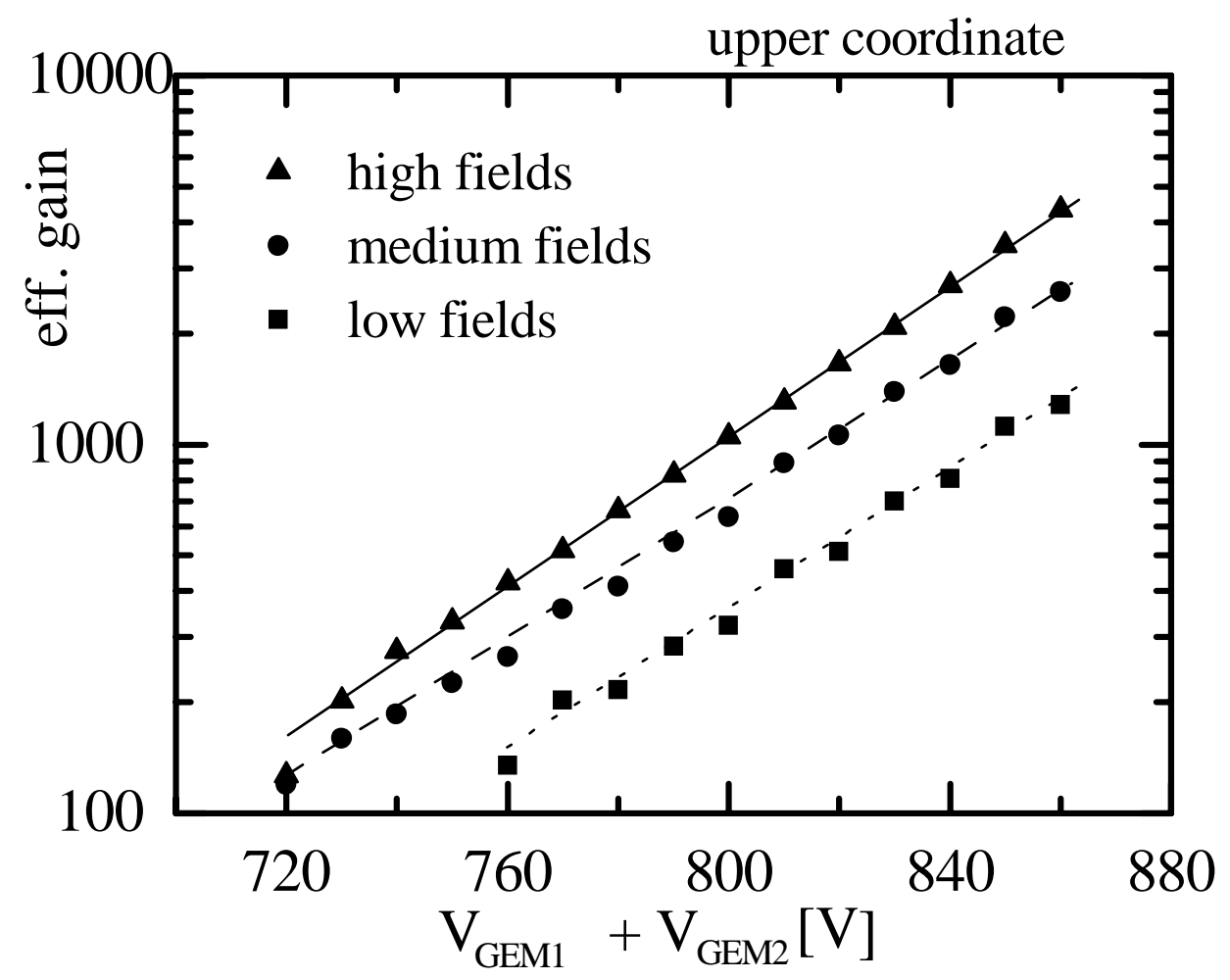

Figure 4.

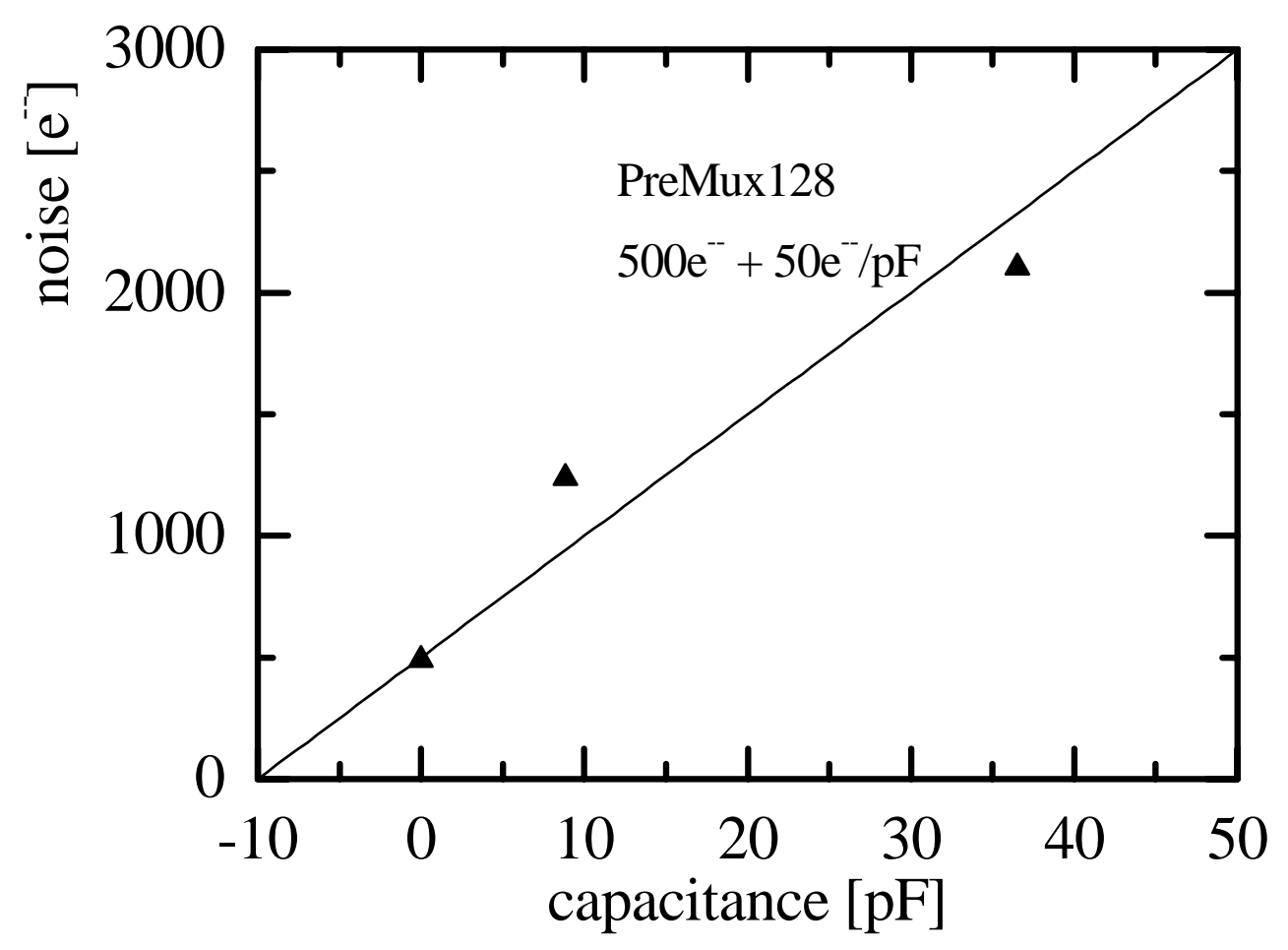

Figure 5. 
COMPASS prototype

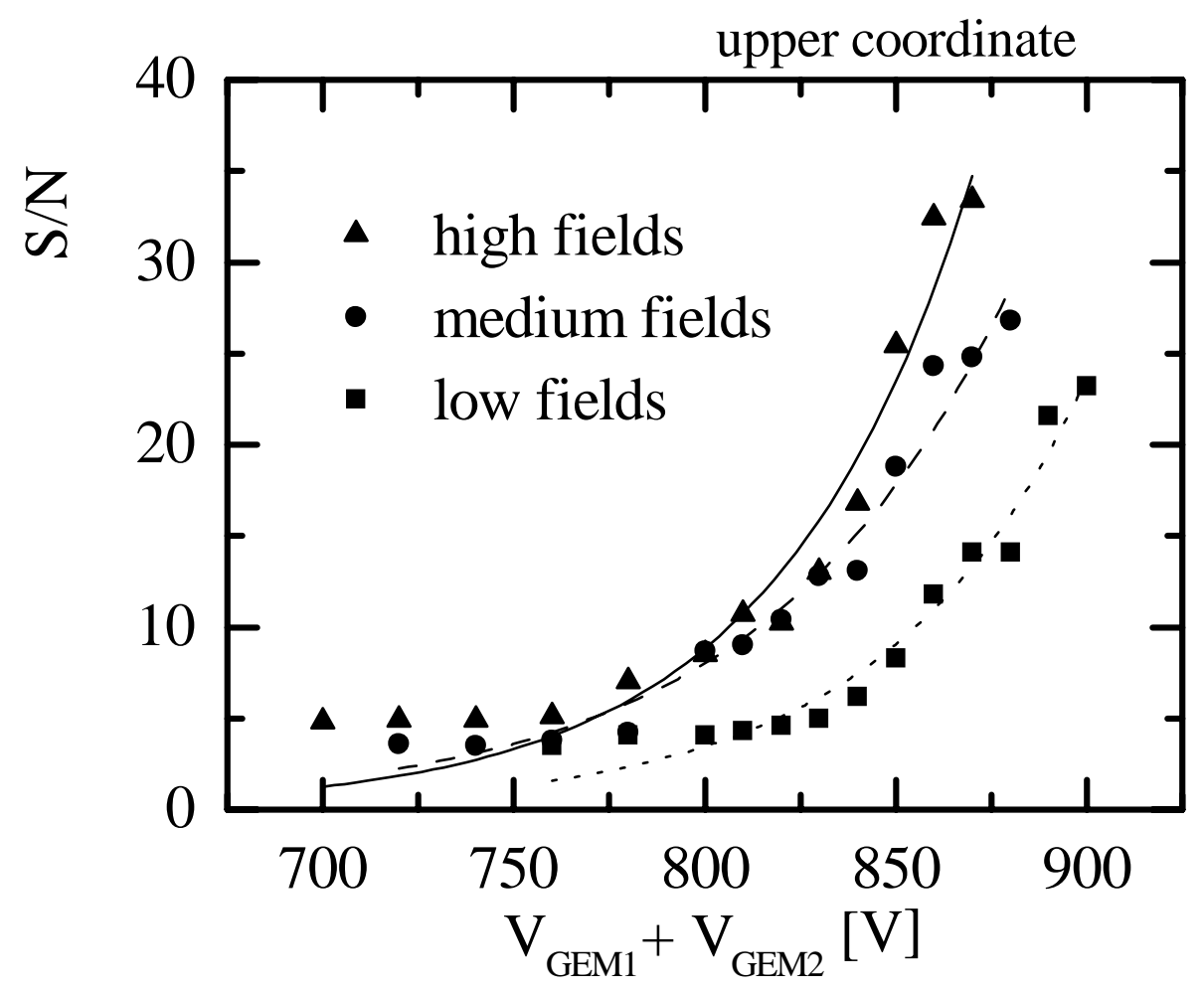

Figure 6 .

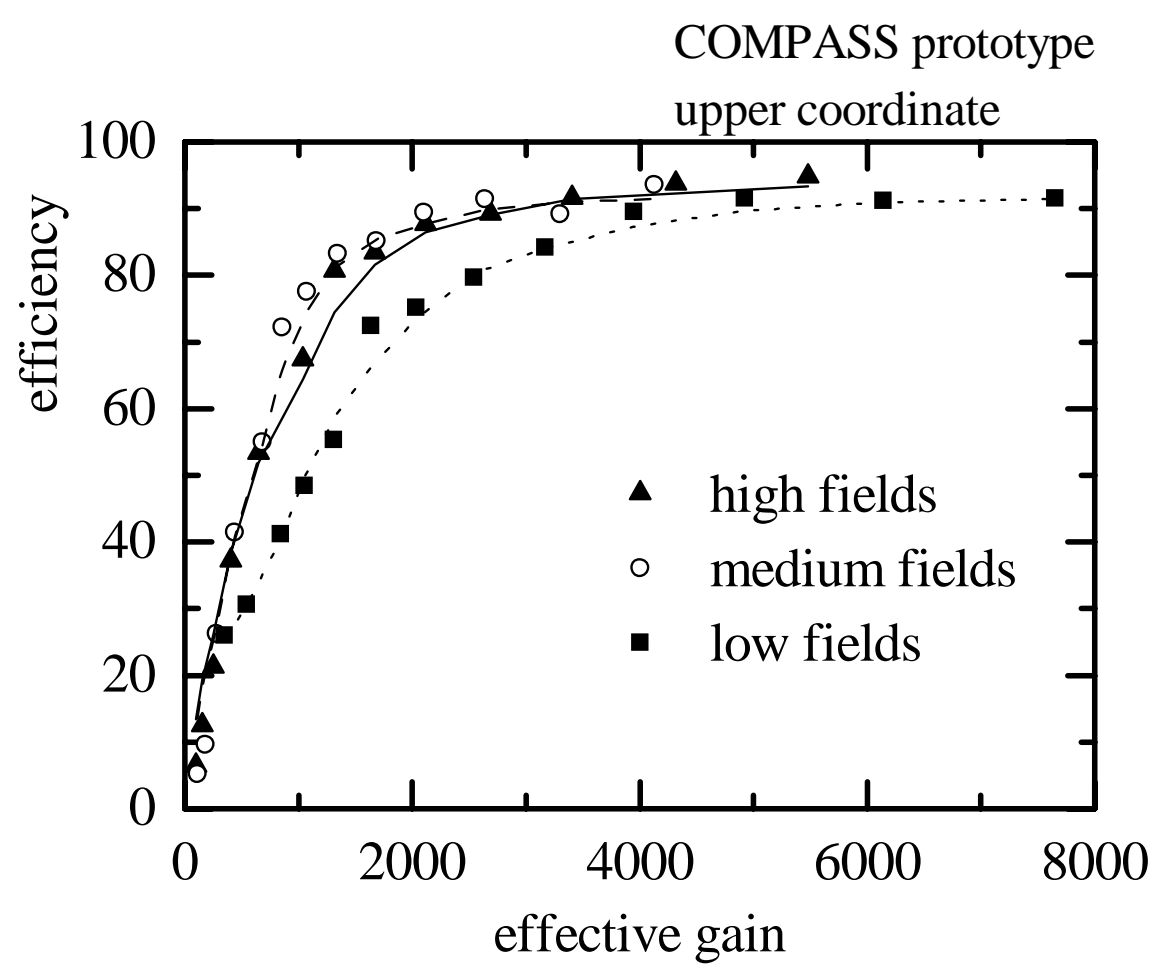

Figure 7. 
a)

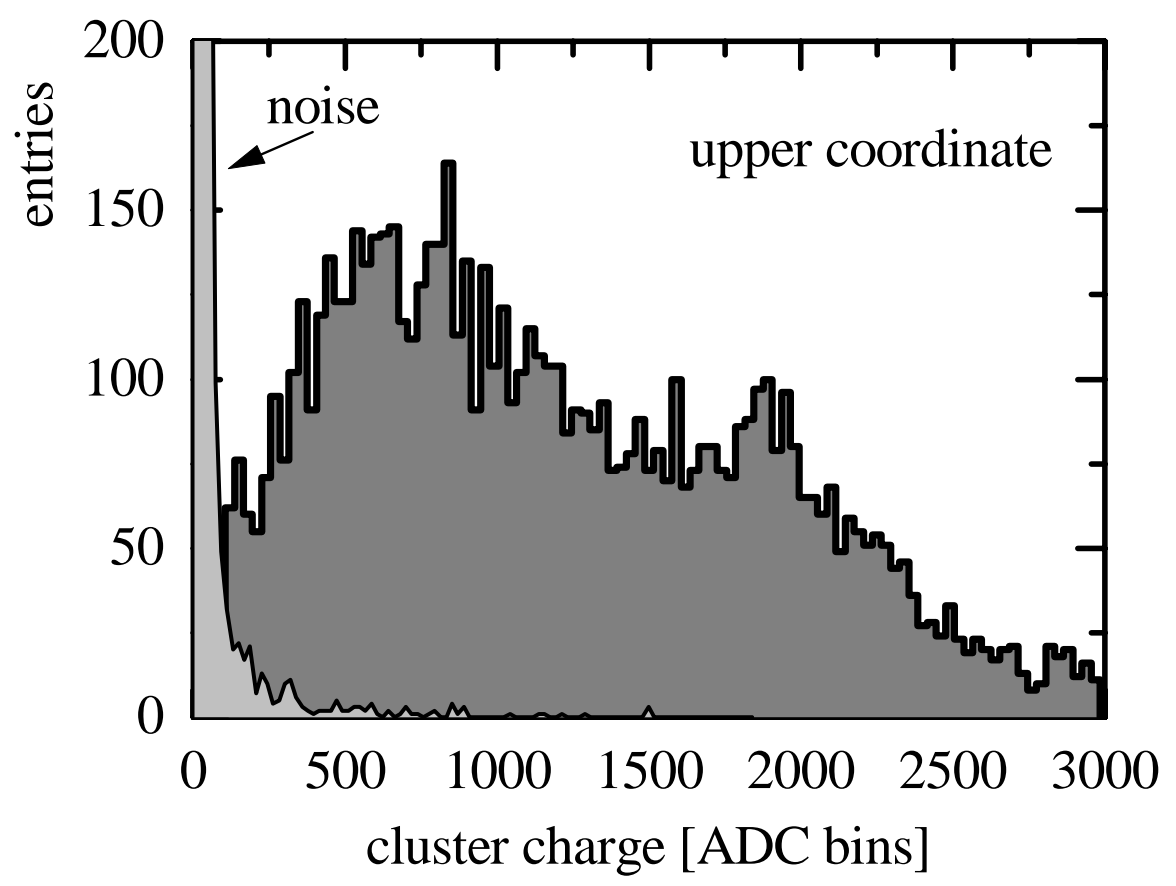

b)

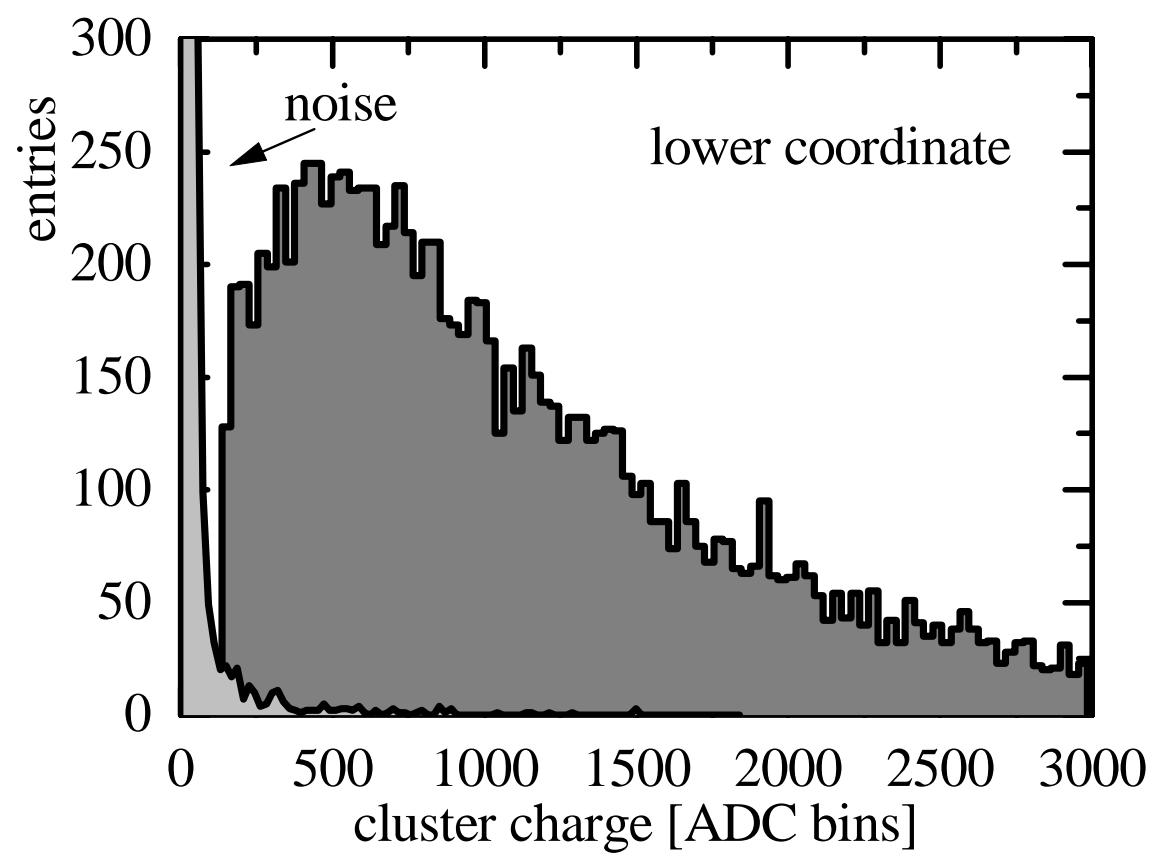

Figure 8. 
a)

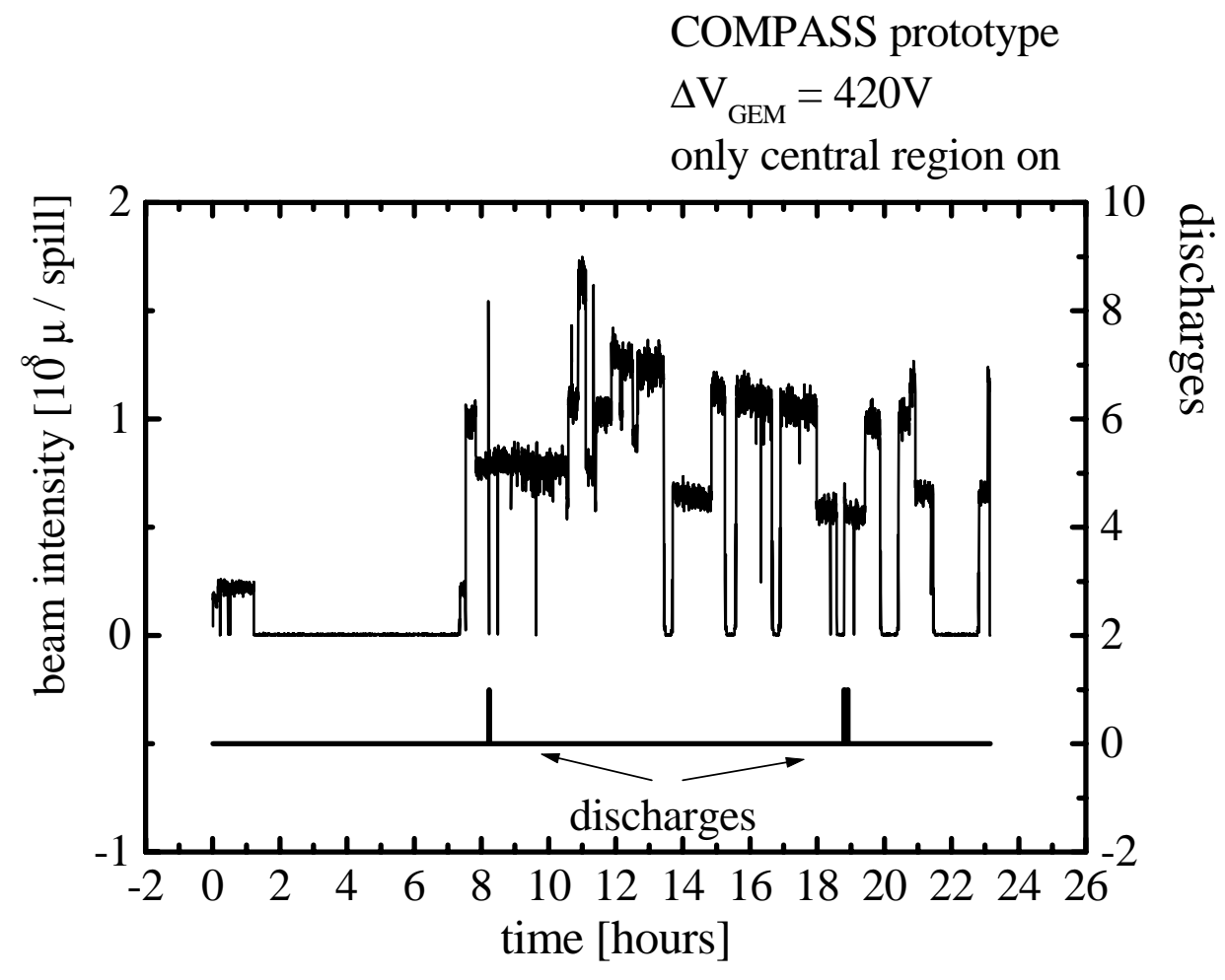

b)

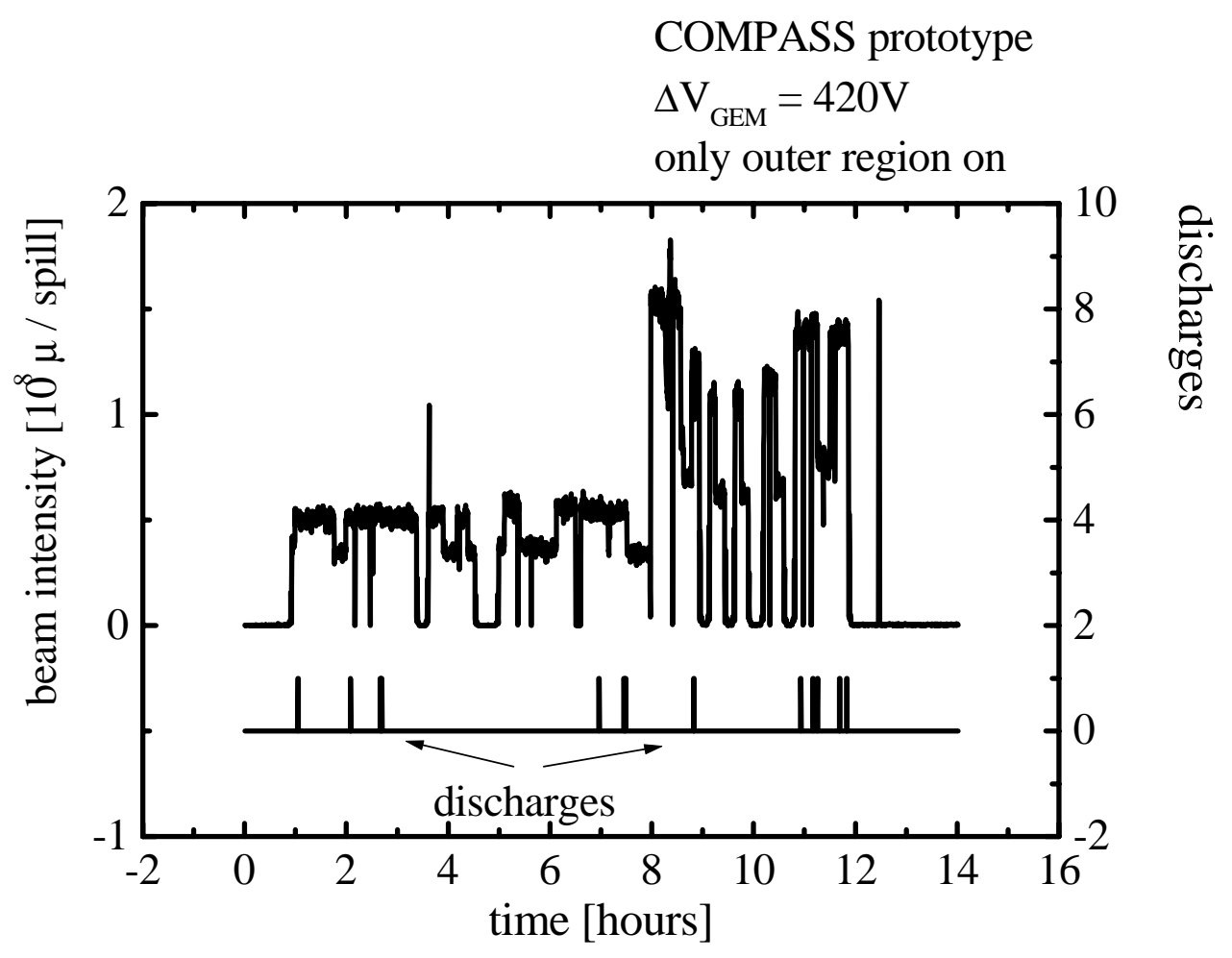

Figure 9. 
a)

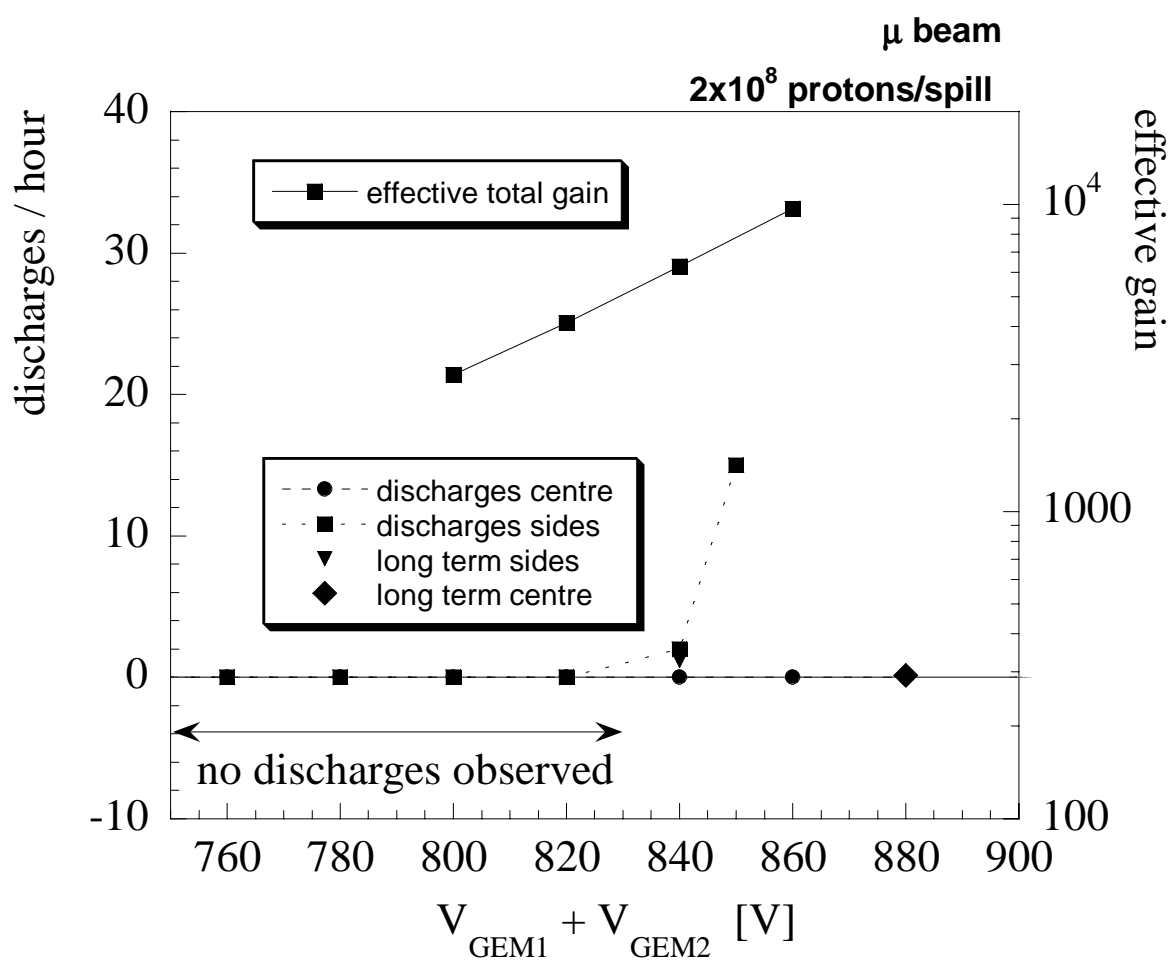

b)

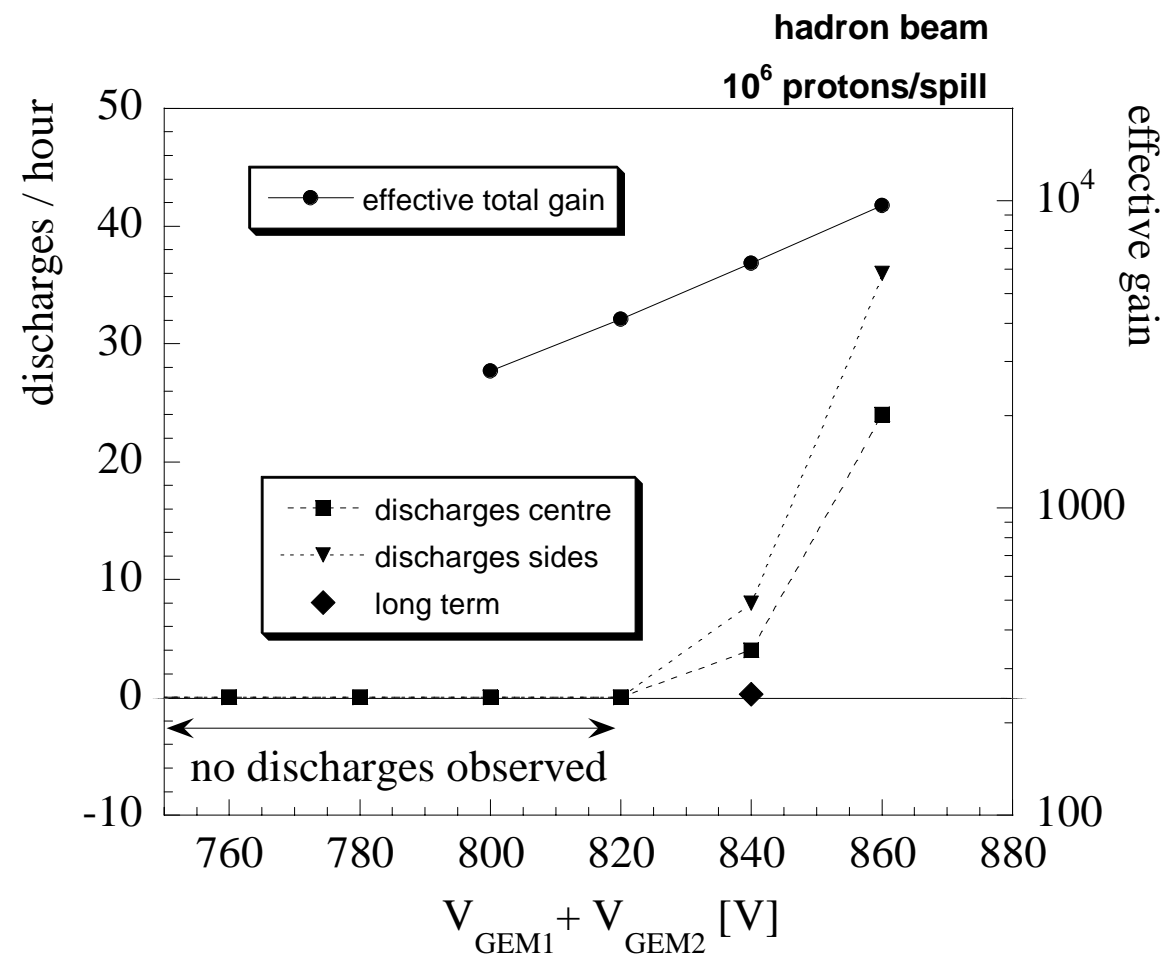

Figure 10. 
a)

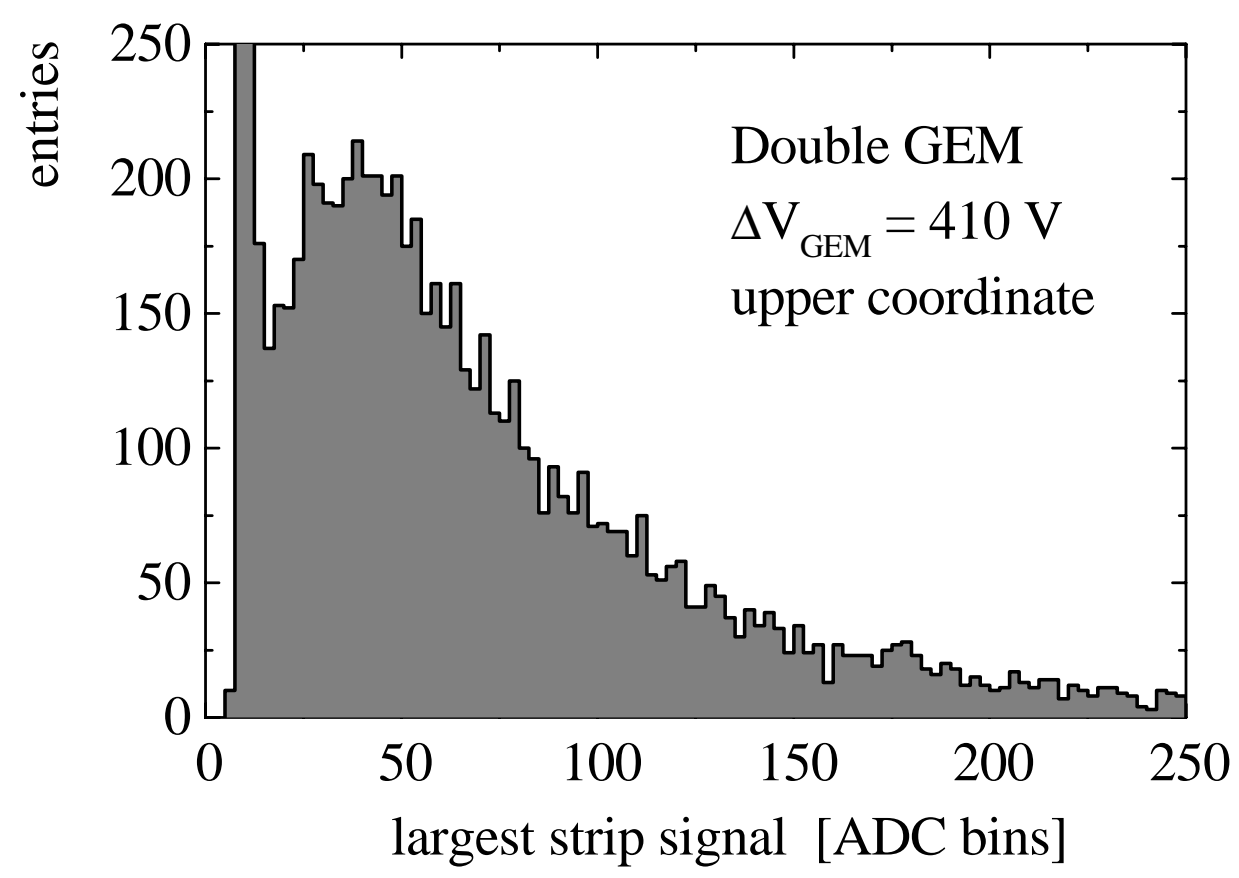

b)

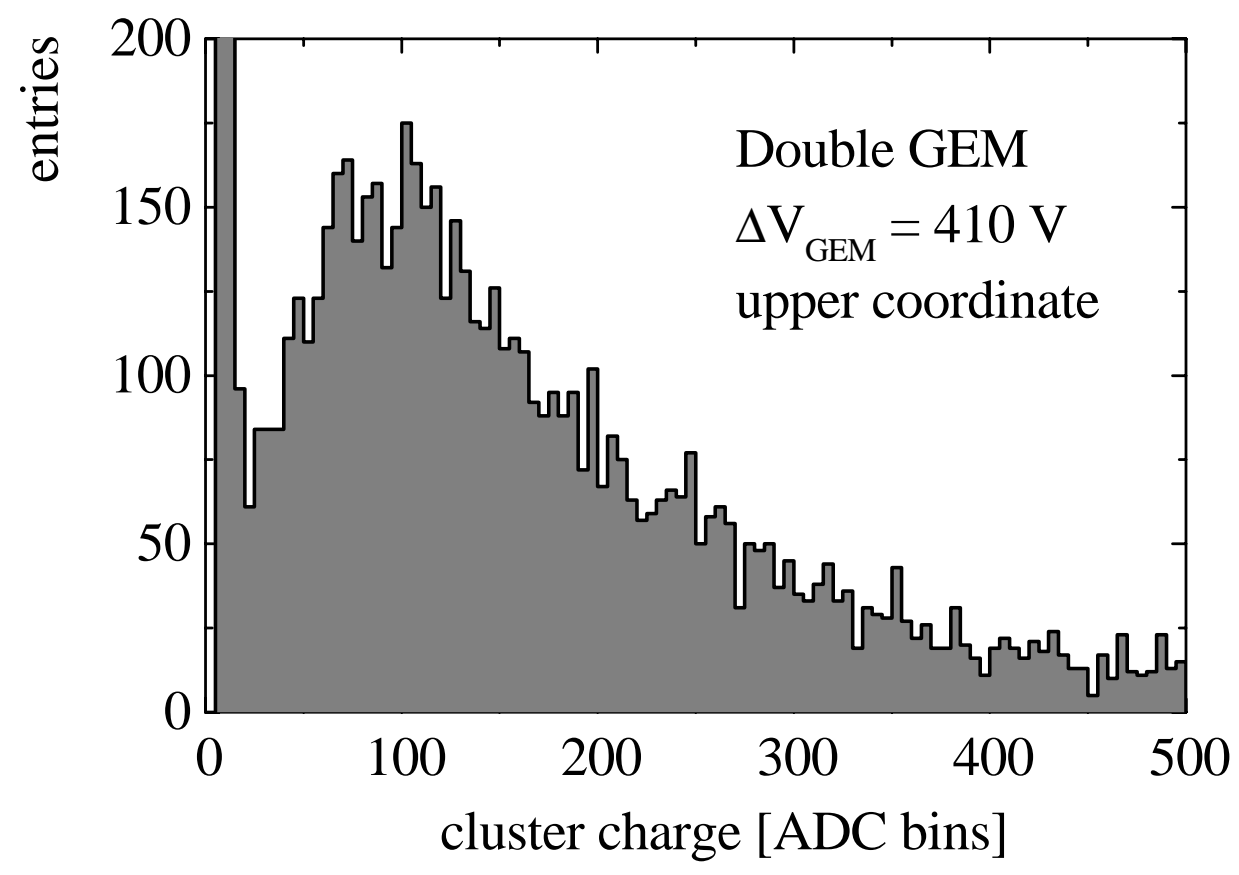

Figure 11. 
a)

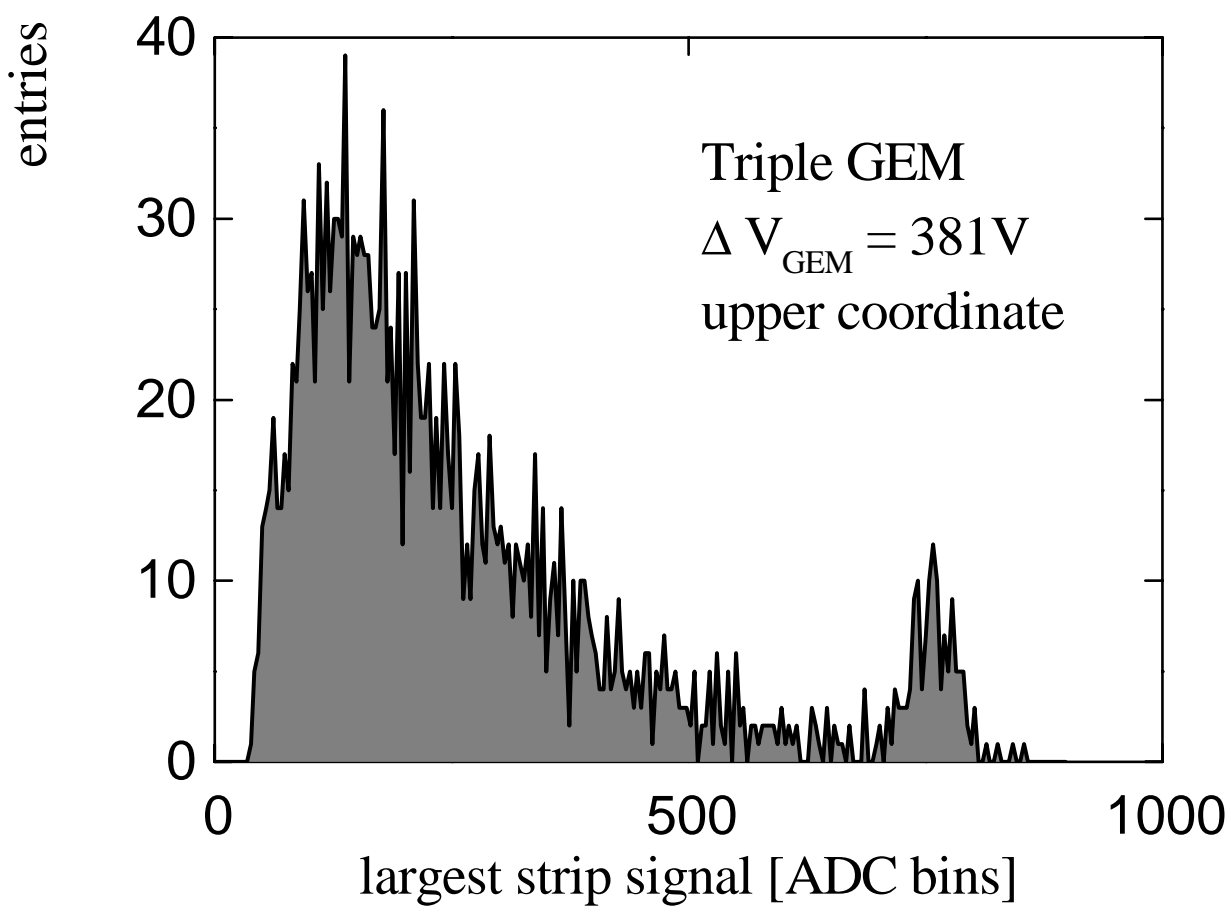

b)

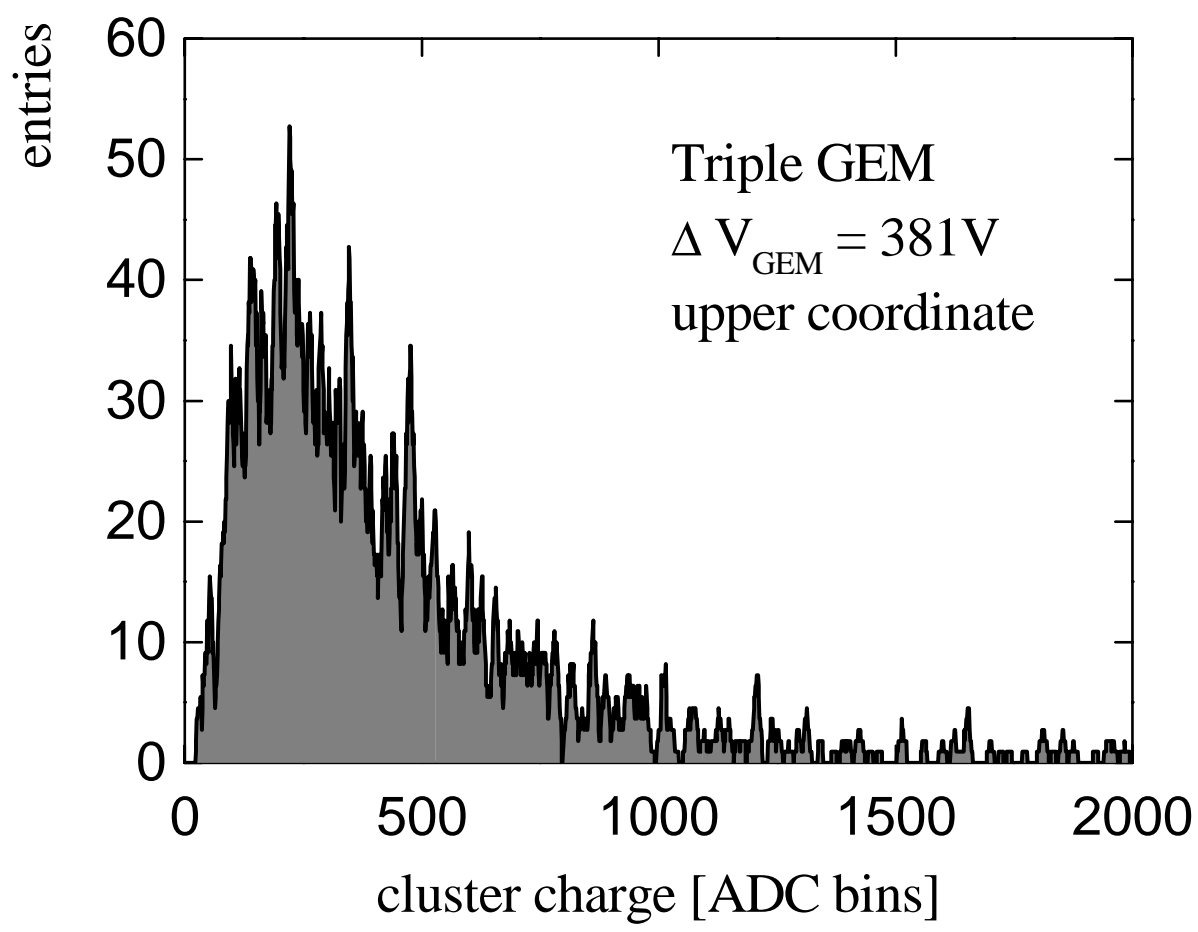

Figure 12. 
a)

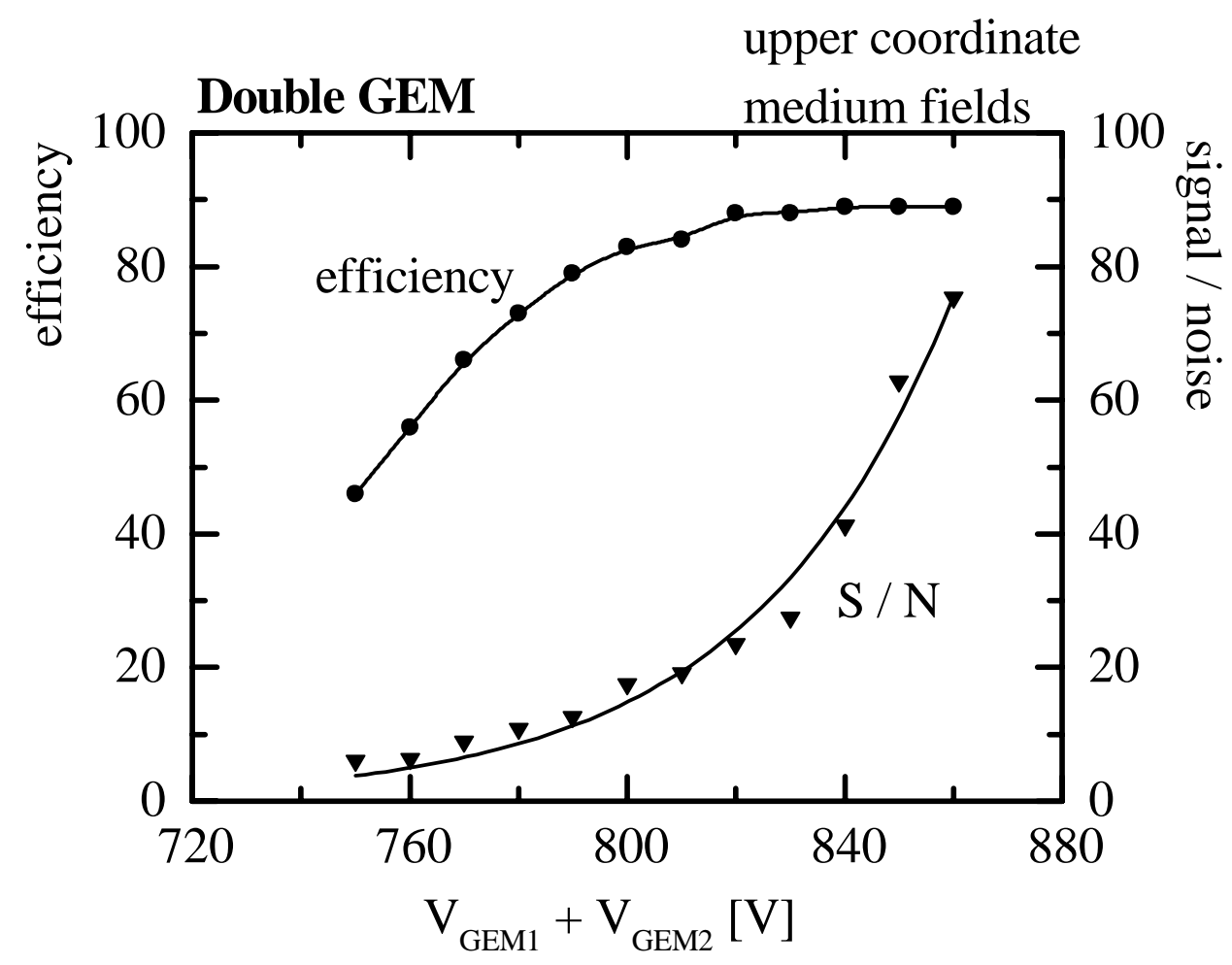

b)

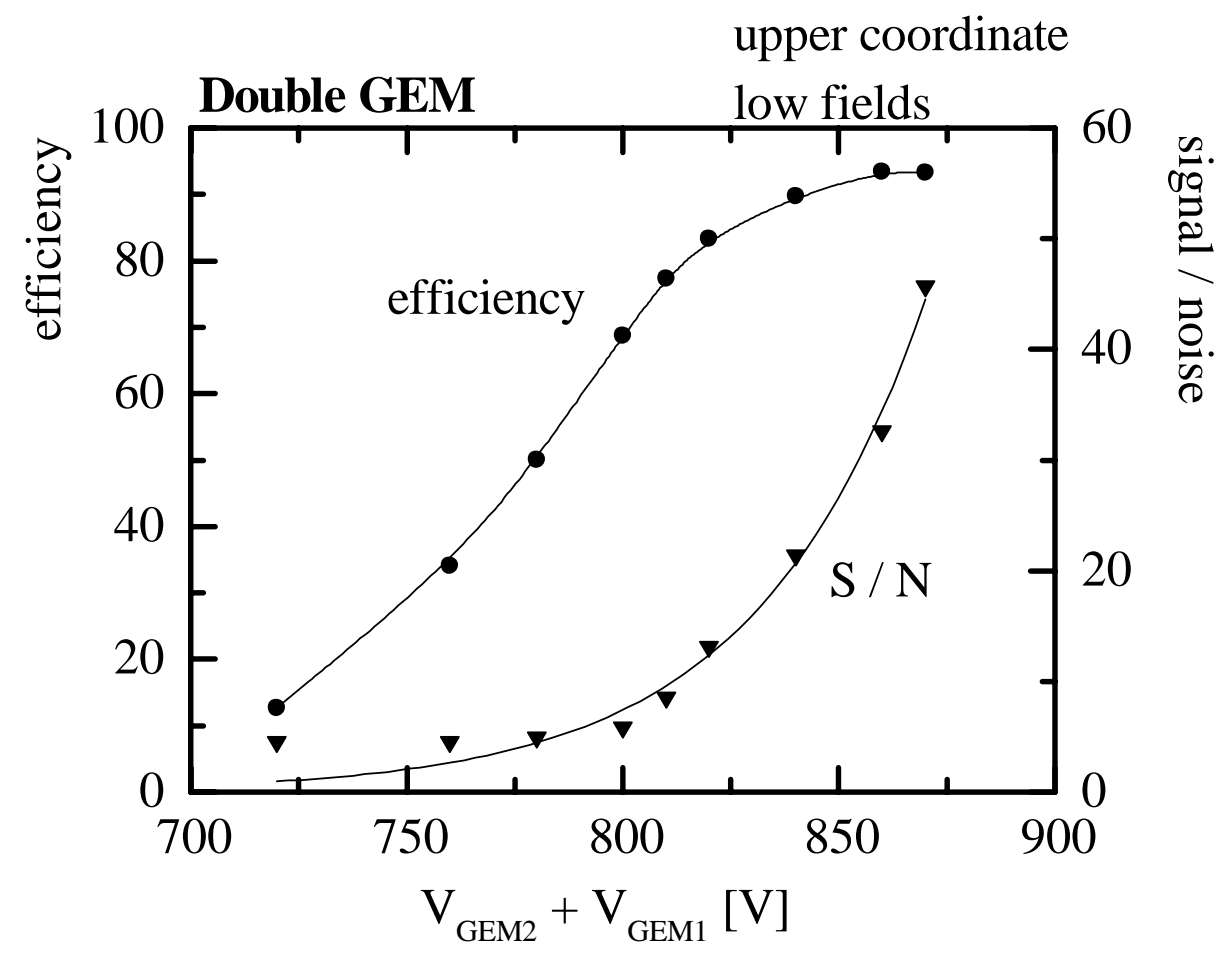

Figure 13. 


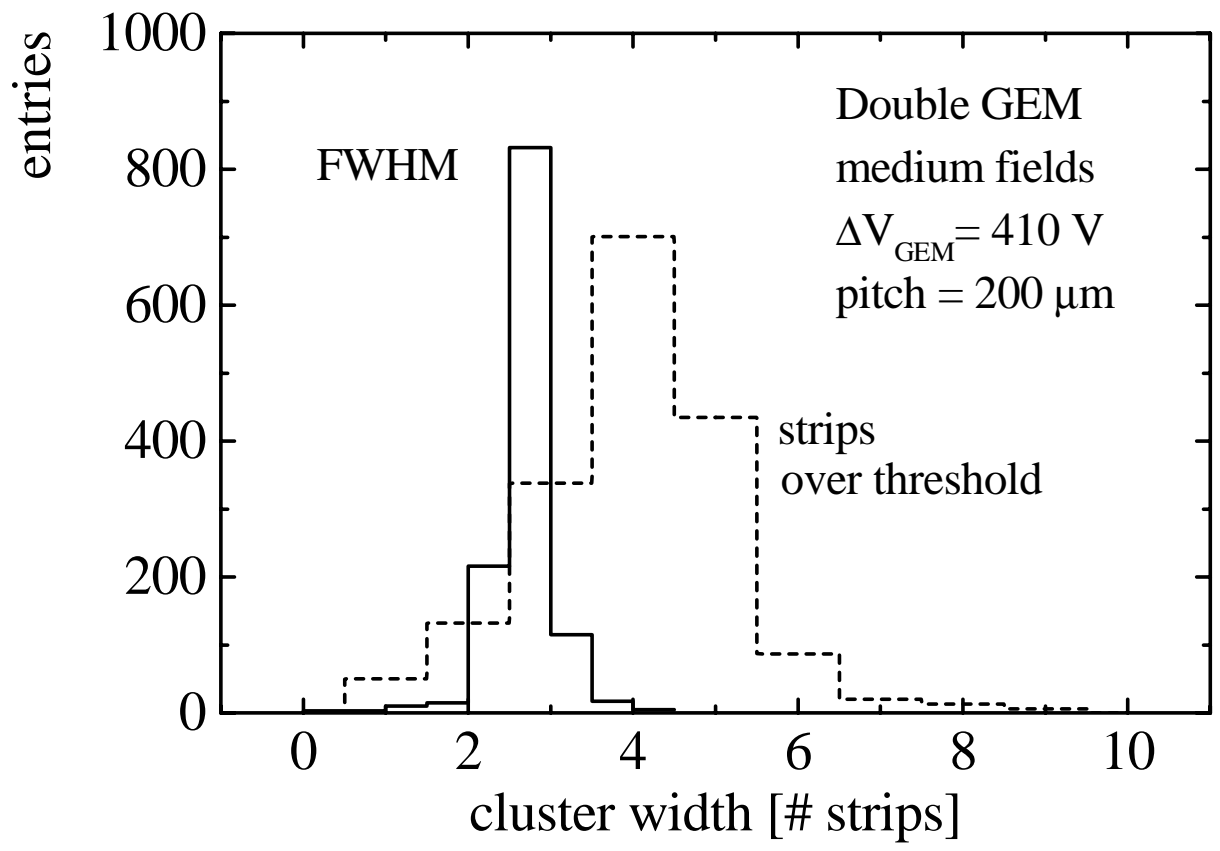

Figure 14.

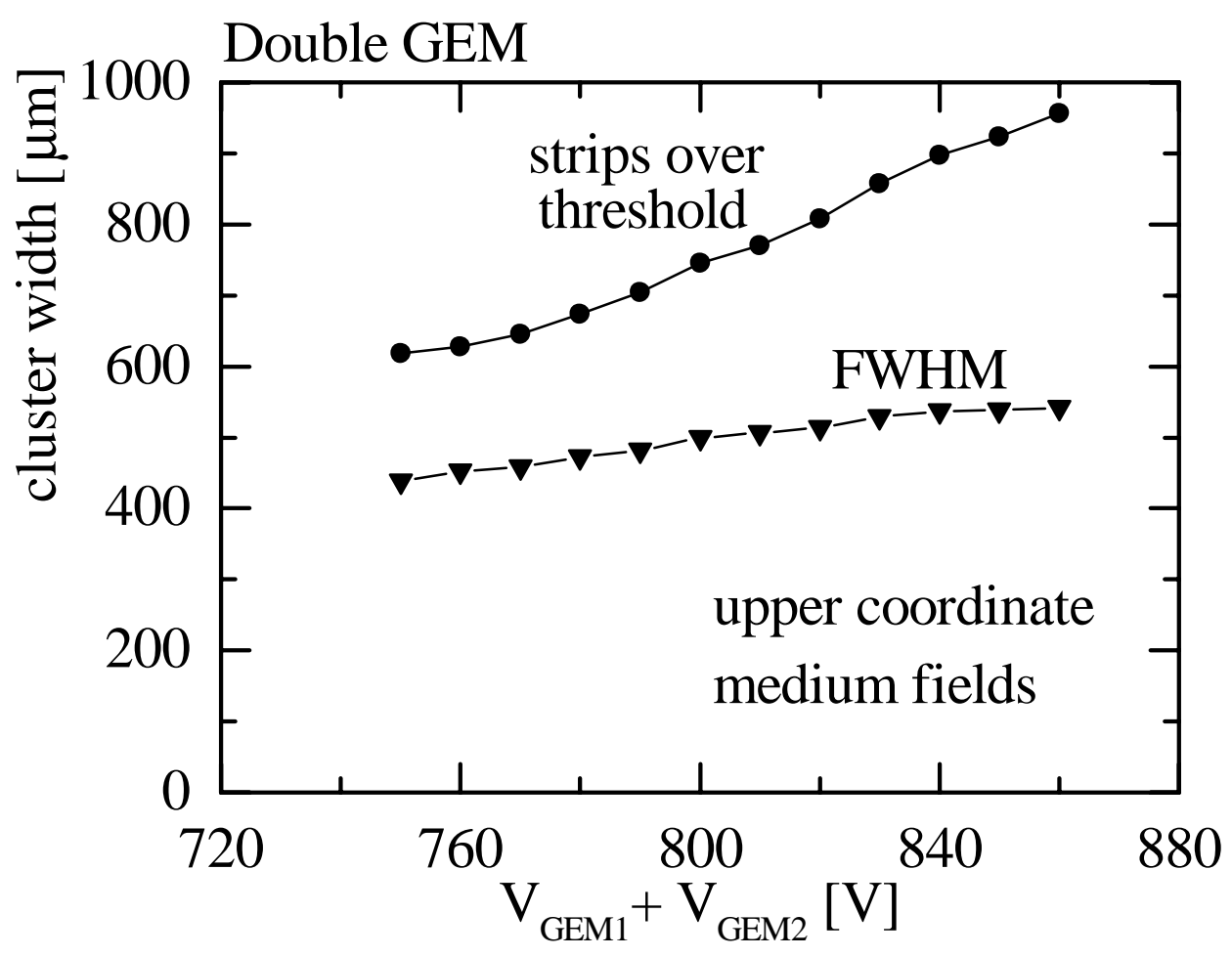

Figure 15. 


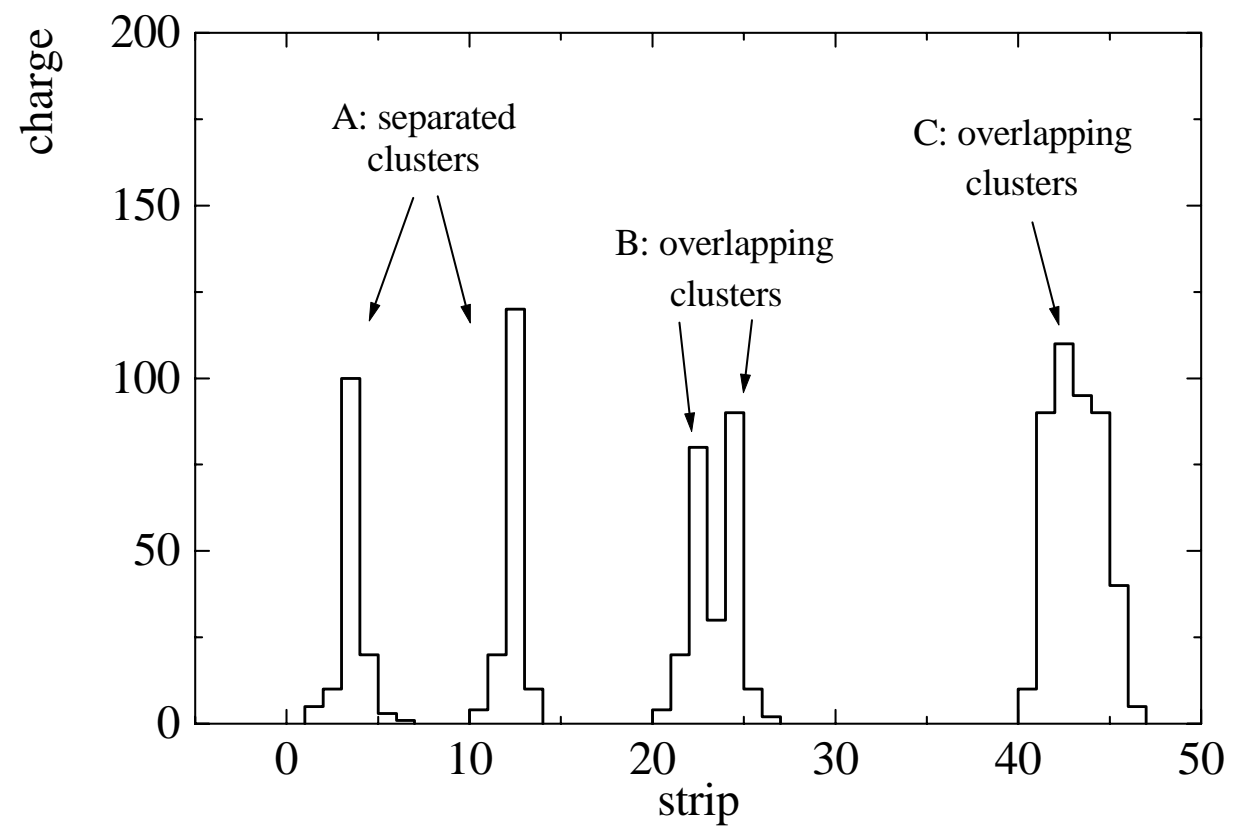

Figure 16.

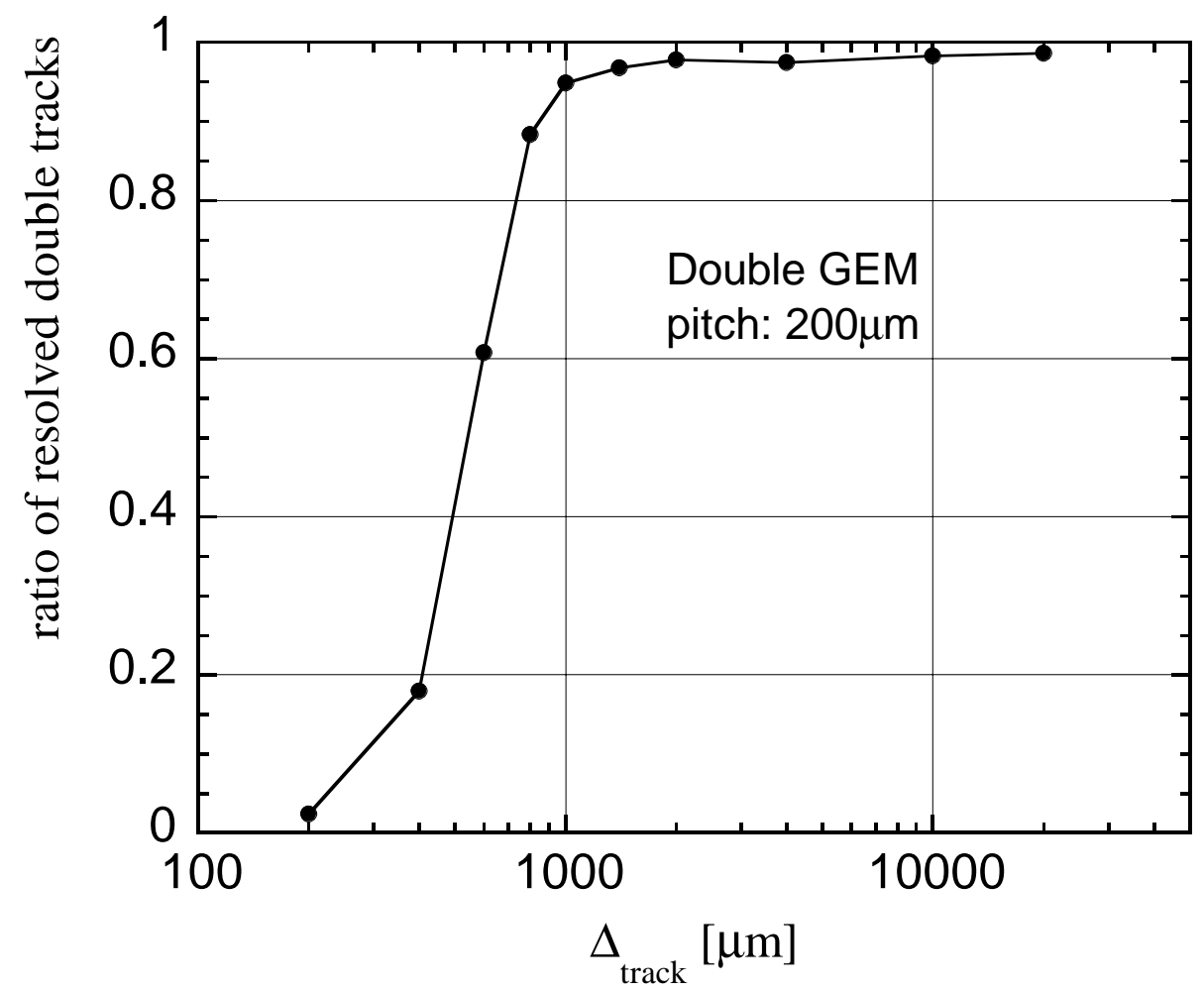

Figure 17. 
a)

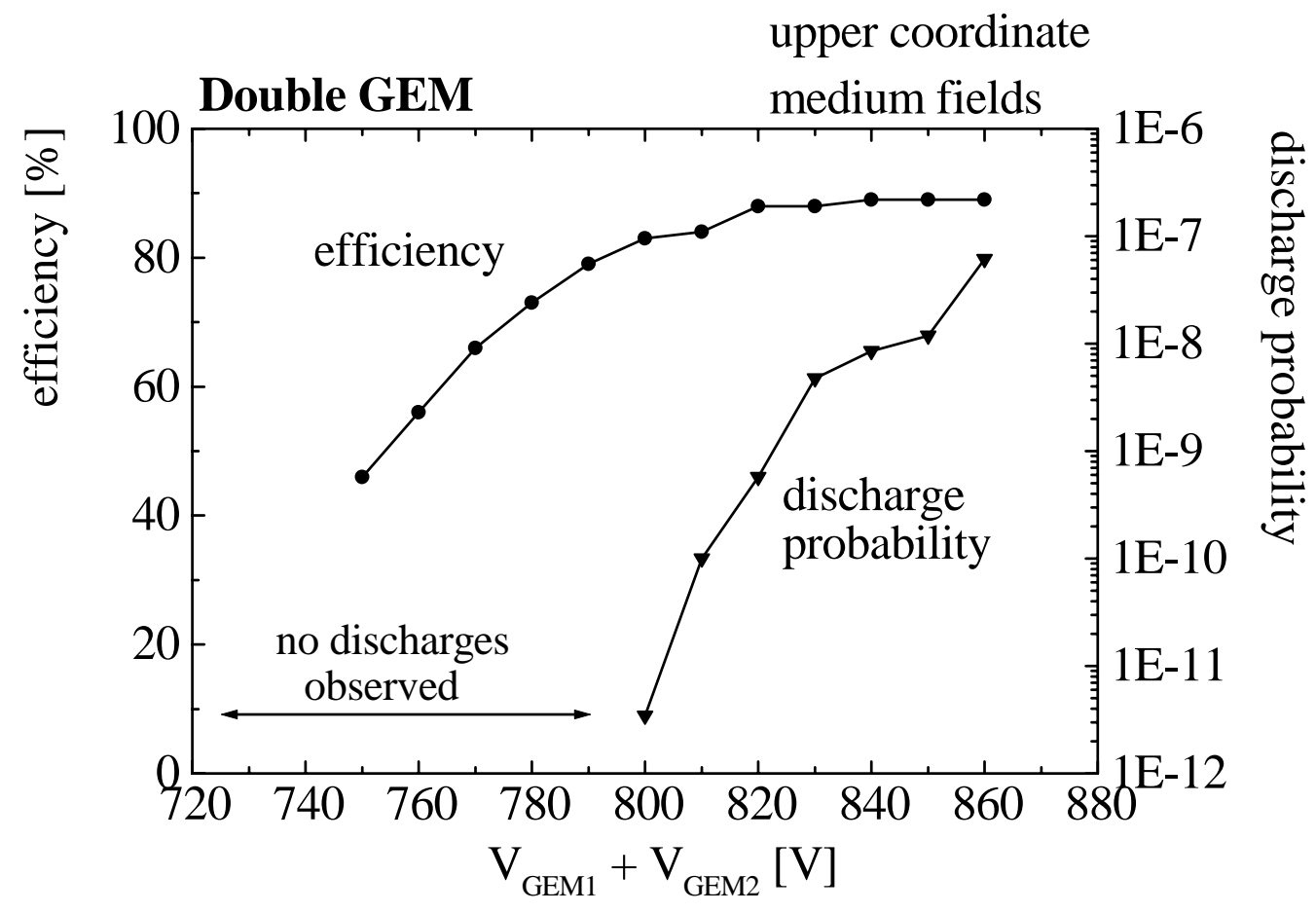

b)

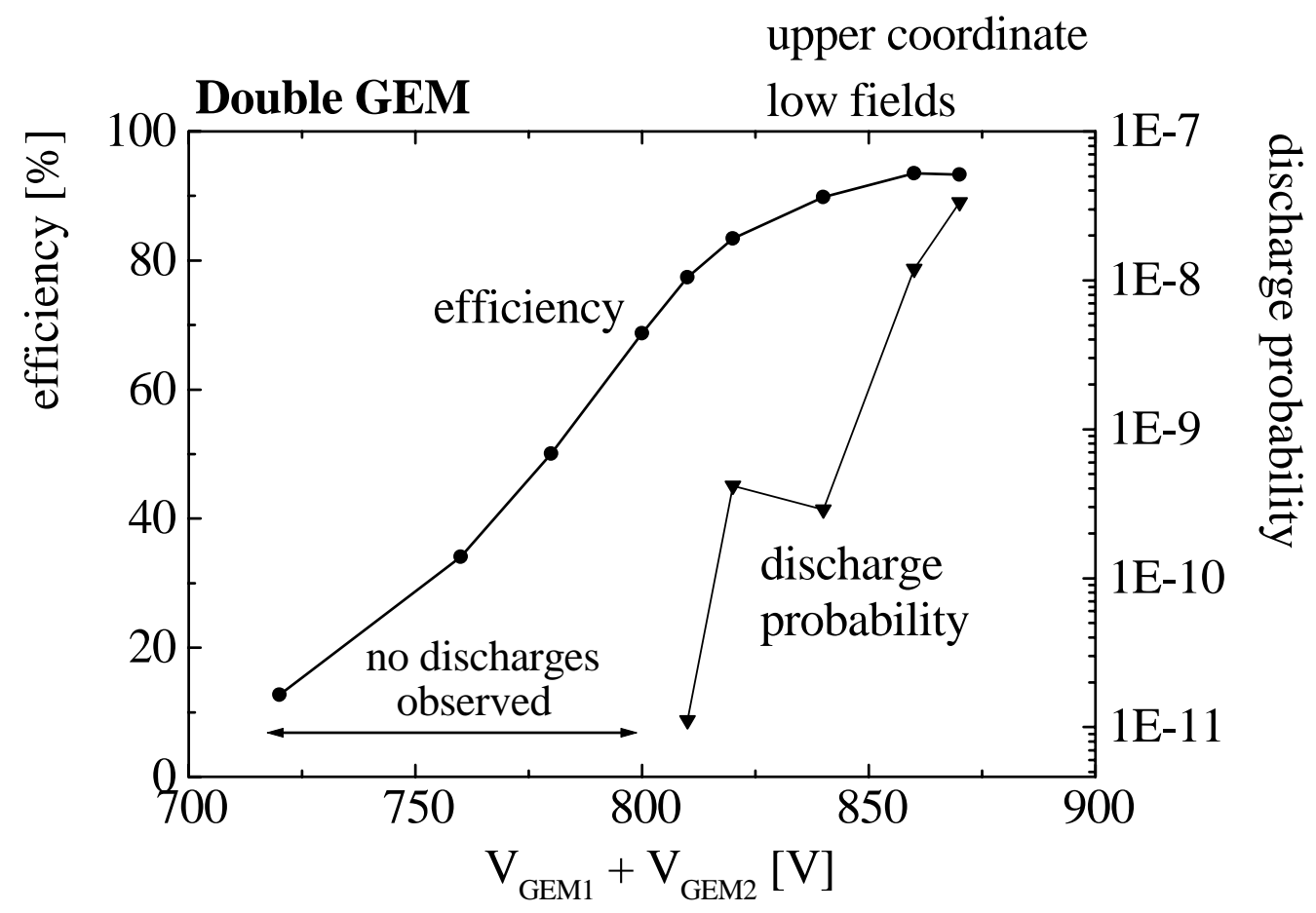

Figure 18. 


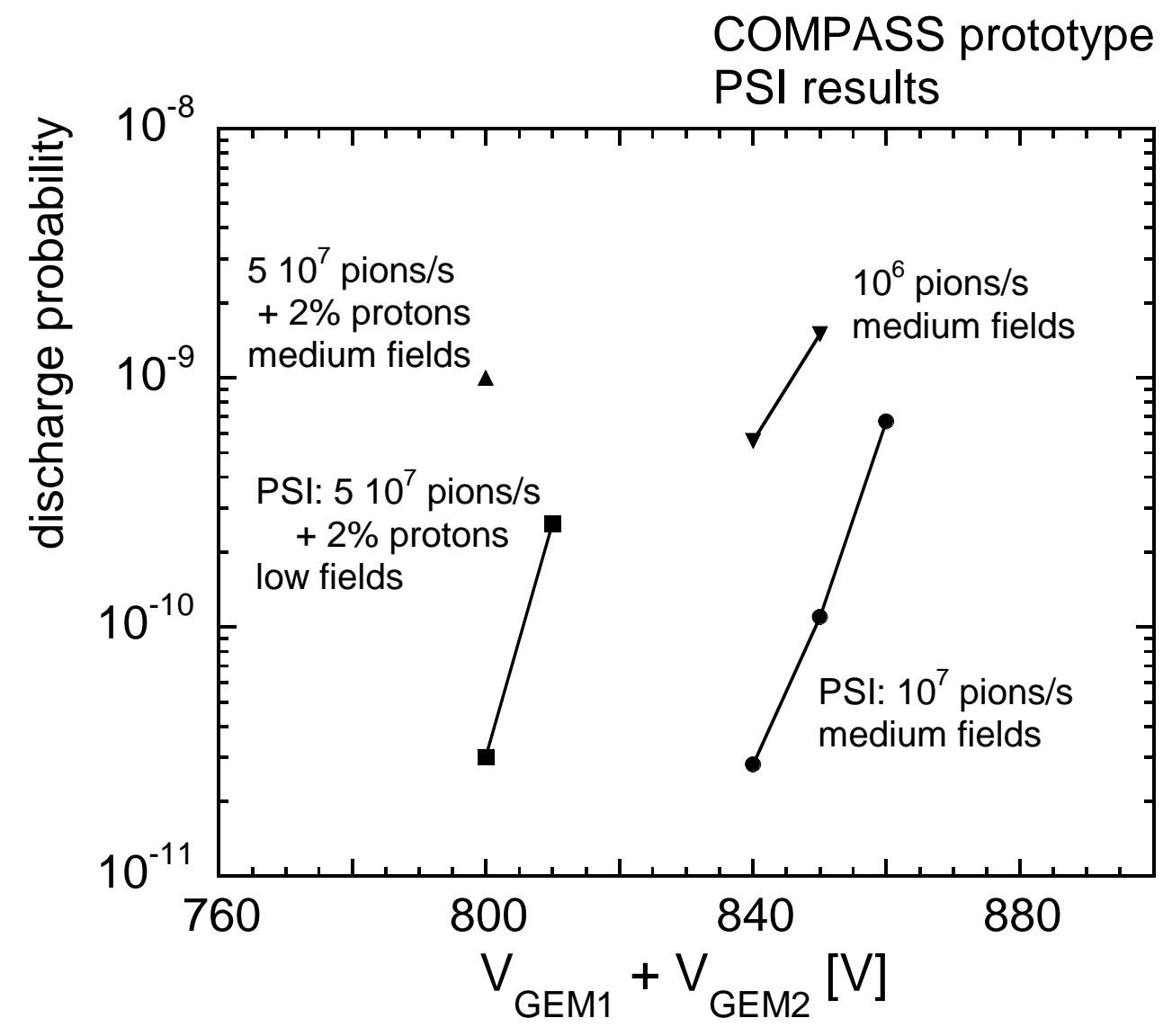

Figure 19. 
a)

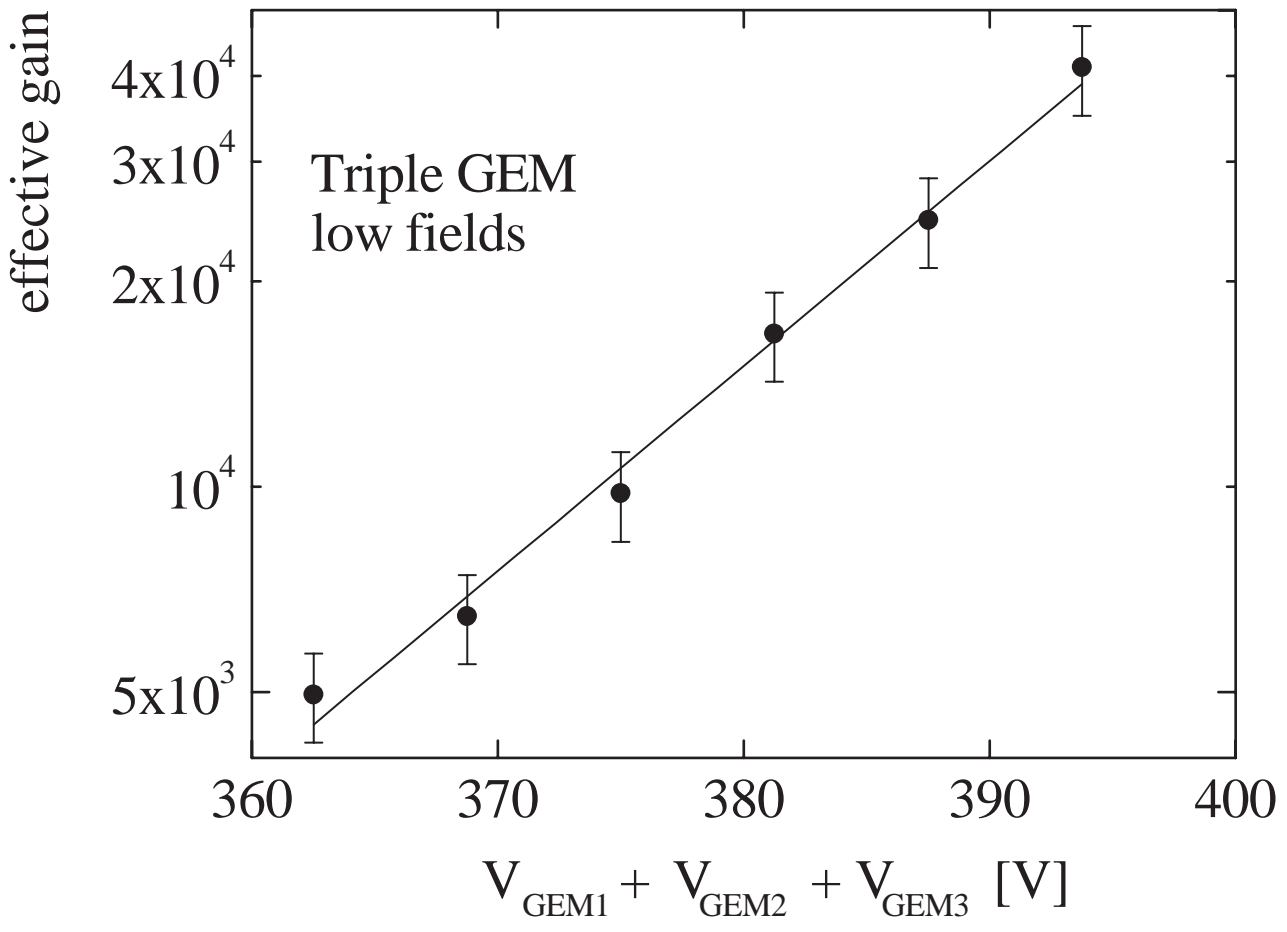

b)

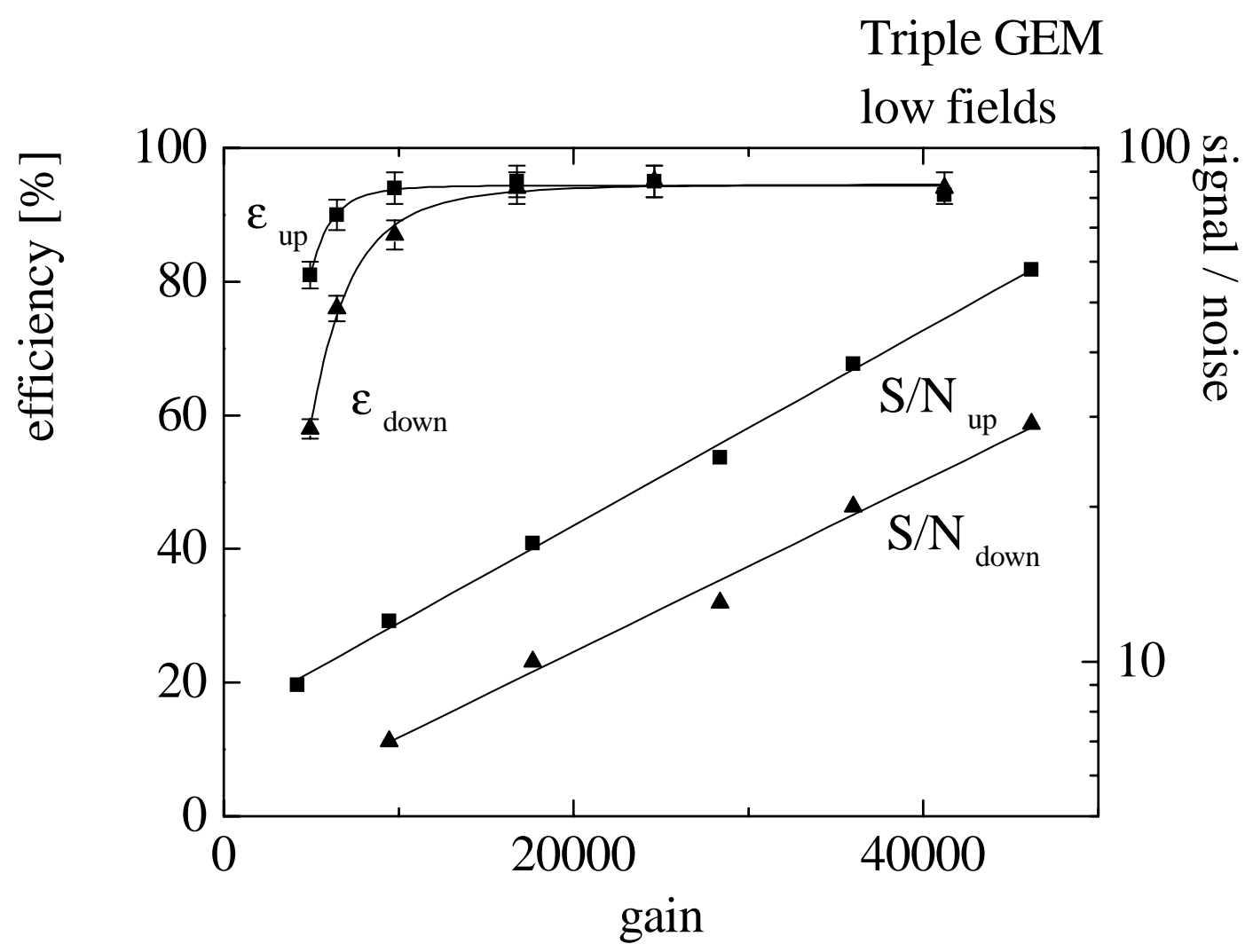

Figure 20. 


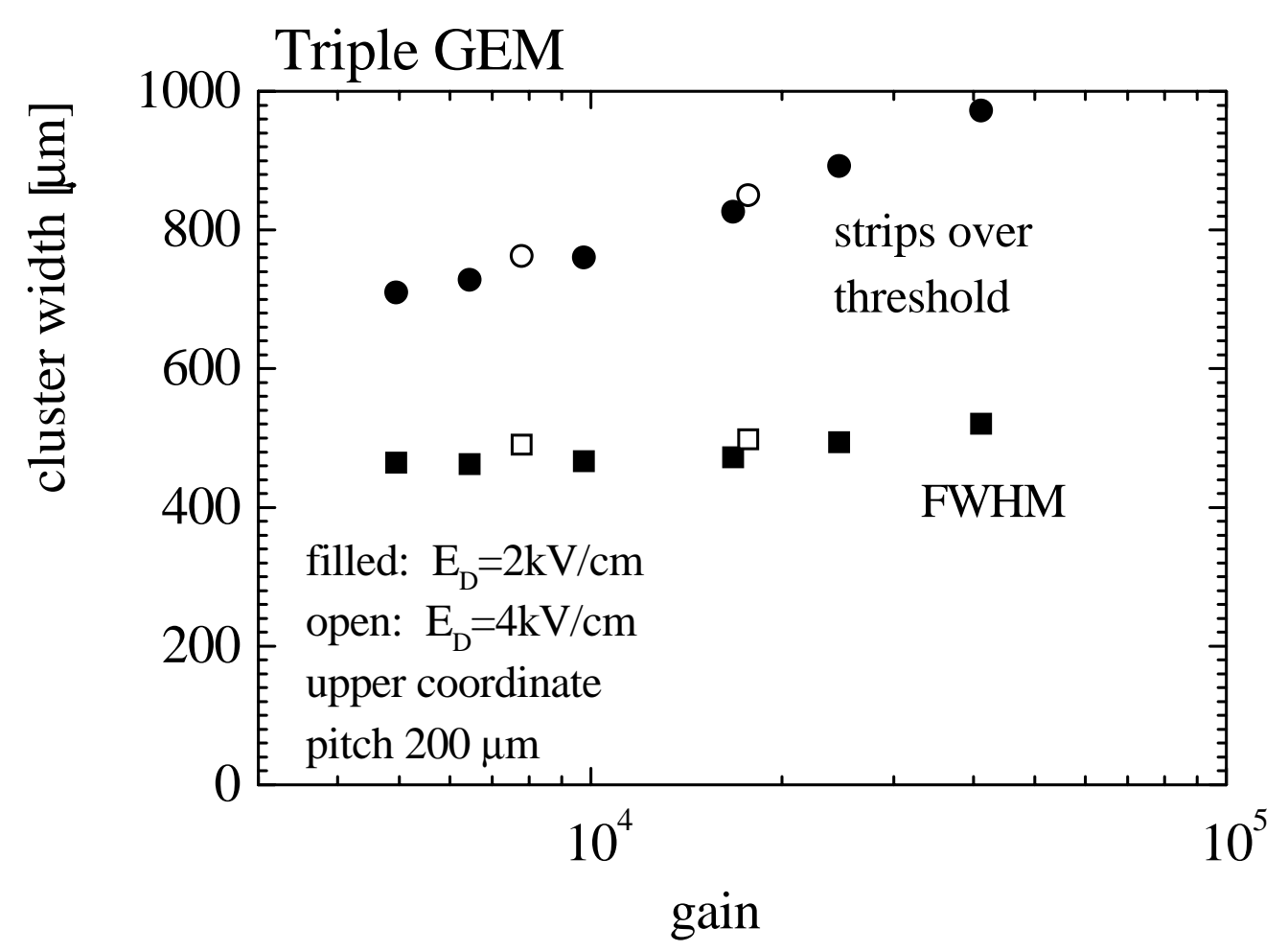

Figure 21.

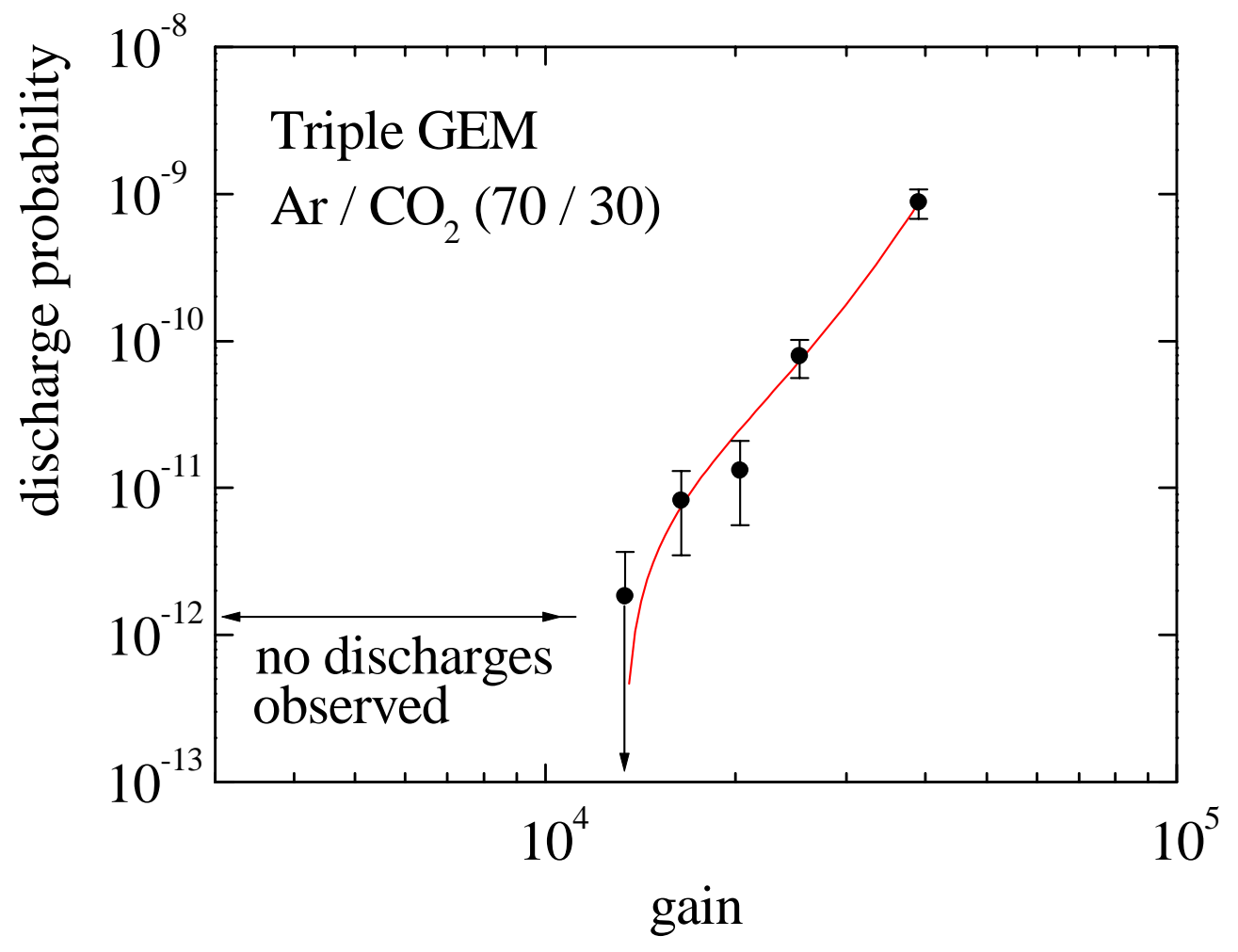

Figure 22. 
a)

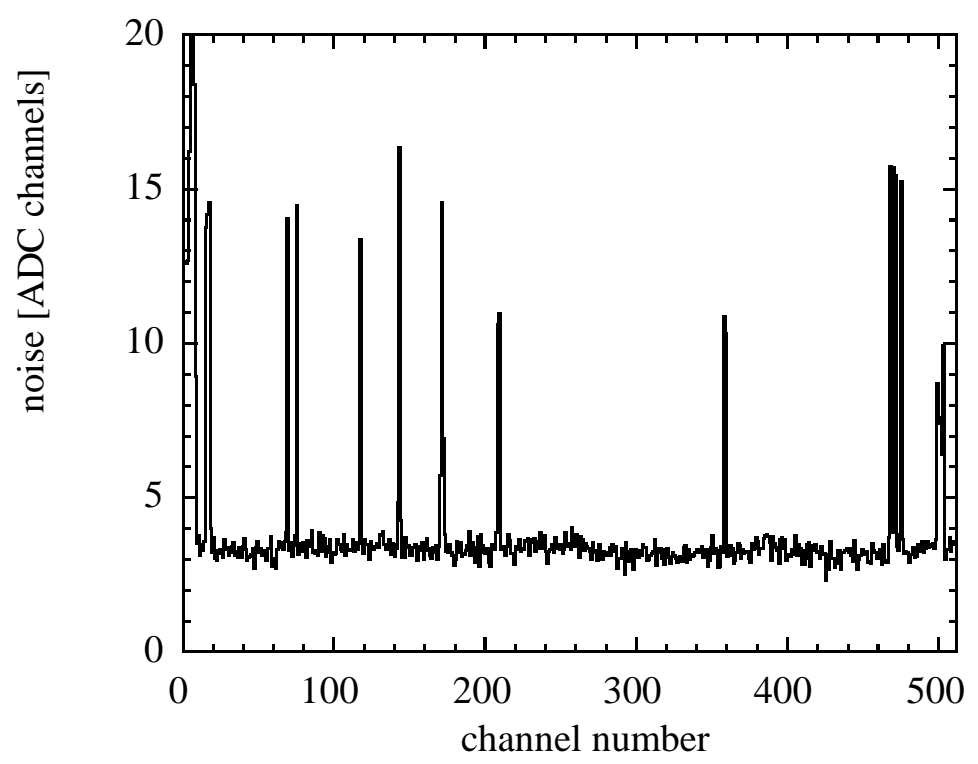

b)

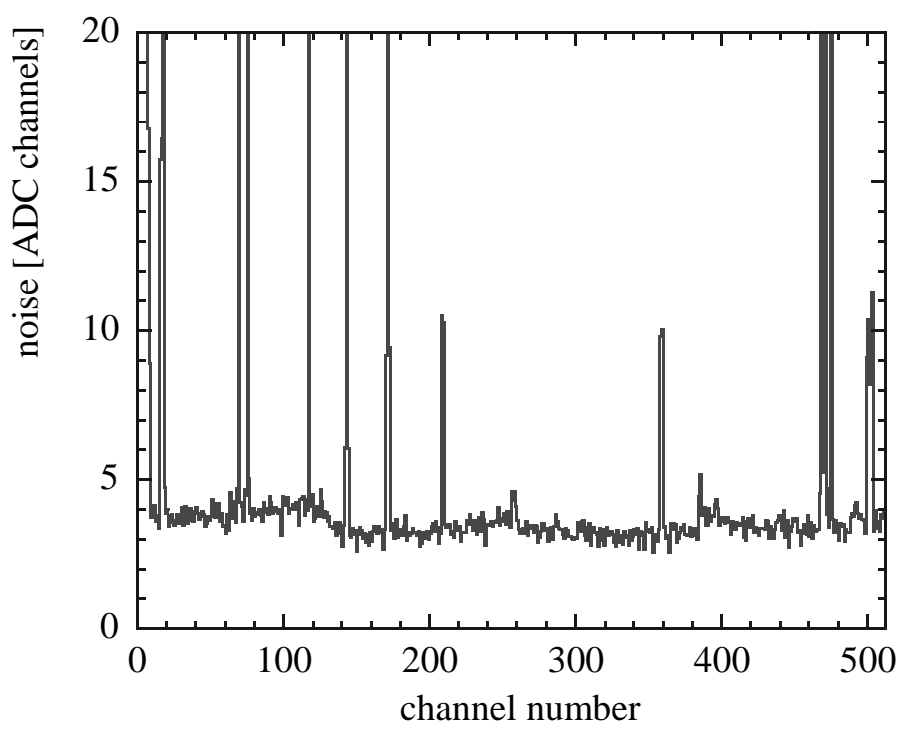

c)

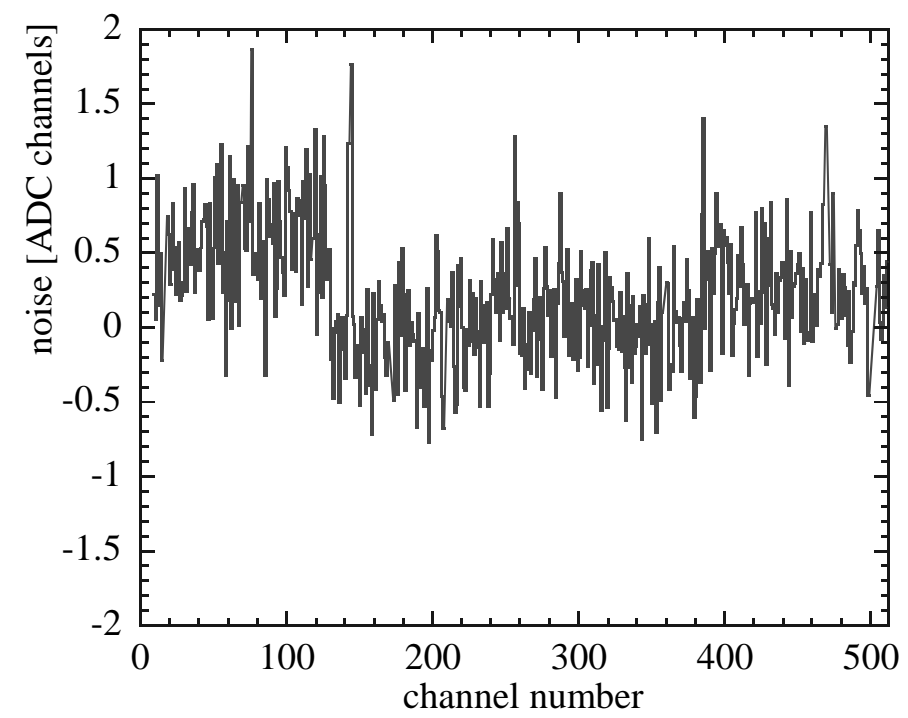

Figure 23. 